\title{
Clathrin Isoform CHC22, a Component of Neuromuscular and Myotendinous Junctions, Binds Sorting Nexin 5 and Has Increased Expression during Myogenesis and Muscle Regeneration $\square$
}

\author{
Mhairi C. Towler, ${ }^{*}$ Paul A. Gleeson, ${ }^{+}$Sachiko Hoshino, ${ }^{\ddagger}$ Paavo Rahkila, $\$$ \\ Venus Manalo, ${ }^{*}$ Norio Ohkoshi, ${ }^{\ddagger}$ Charles Ordahl," Robert G. Parton, ${ }^{\text {II }}$ and \\ Frances M. Brodsky*\#
}

\begin{abstract}
*The G.W. Hooper Foundation, Department of Microbiology and Immunology and Departments of Biopharmaceutical Sciences and Pharmaceutical Chemistry, University of California, San Francisco, California 94143-0552; ${ }^{\text {T}}$ The Russell Grimwade School of Biochemistry and Molecular Biology, University of Melbourne, Victoria 3010, Melbourne, Australia; ‘Department of Neurology, Institute of Clinical Medicine, University of Tsukuba, Tsukuba City, Japan; §Kuusitie 67, Fin-40800 Vaajakoski, Finland; "Department of Anatomy and Cardiovascular Research Institute, University of California, San Francisco, California, 94143-0452; and IInstitute for Molecular Biosciences, Center for Microscopy and Microanalysis, and School of Biomedical Sciences, University of Queensland, Brisbane 4072, Australia
\end{abstract}

Submitted March 23, 2004; Revised April 23, 2004; Accepted April 26, 2004

Monitoring Editor: Juan S. Bonifacino

\begin{abstract}
The muscle isoform of clathrin heavy chain, $\mathrm{CHC} 22$, has $85 \%$ sequence identity to the ubiquitously expressed $\mathrm{CHC} 17$, yet its expression pattern and function appear to be distinct from those of well-characterized clathrin-coated vesicles. In mature muscle $\mathrm{CHC} 22$ is preferentially concentrated at neuromuscular and myotendinous junctions, suggesting a role at sarcolemmal contacts with extracellular matrix. During myoblast differentiation, CHC22 expression is increased, initially localized with desmin and nestin and then preferentially segregated to the poles of fused myoblasts. CHC22 expression is also increased in regenerating muscle fibers with the same time course as embryonic myosin, indicating a role in muscle repair. CHC22 binds to sorting nexin 5 through a coiled-coil domain present in both partners, which is absent in $\mathrm{CHC} 17$ and coincides with the region on $\mathrm{CHC17}$ that binds the regulatory light-chain subunit. These differential binding data suggest a mechanism for the distinct functions of $\mathrm{CHC} 22$ relative to $\mathrm{CHC} 17$ in membrane traffic during muscle development, repair, and at neuromuscular and myotendinous junctions.
\end{abstract}

\section{INTRODUCTION}

Skeletal muscle is composed of highly organized multinuclear cells or myofibers. Their internal organization is dictated by the arrangement of actin and myosin myofibrils with associated protein components, the former being anchored at regular intervals in Z-disks, for force generation during muscle contraction (Clark et al., 2002). In addition to the basic mechanical components, muscle function depends on a number of specialized and housekeeping membrane compartments and membrane traffic pathways (for review

Article published online ahead of print. Mol. Biol. Cell 10.1091/ mbc.E04-03-0249. Article and publication date are available at www.molbiolcell.org/cgi/doi/10.1091/mbc.E04-03-0249.

$\square$ Online version of this article contains supporting material. Online version is available at www.molbiolcell.org.

\# Corresponding author. E-mail address: fmarbro@itsa.ucsf.edu. Abbreviations used: BngTx, bungarotoxin; CCVs, clathrincoated vesicles; $\mathrm{CHC}$, clathrin heavy chain; LC, light chain; ECM, extracellular matrix; GLUT4, glucose transporter 4; MTJ, myotendinous junction; NMJ, neuromuscular junction; PX, phox homology; SNX, sorting nexin. see Towler et al., 2004). These membrane components are also highly organized to coordinate with the contractile apparatus. Muscle integrity further depends on specialized membrane repair pathways to deal with chronic load-bearing stress, and muscle development relies on specialized membrane fusion processes as well as elaborate membrane organization. An emerging theme in muscle cell biology is that muscle-specific versions of ubiquitous membrane traffic components are responsible for aspects of the specialized membrane traffic involved in muscle function. Two examples include isoforms of amphiphysin and caveolin, which have been implicated in T-tubule development in nonhuman species (Parton et al., 1997; Zhang and Zelhof, 2002) and are defective in vertebrate muscle disease (Fischer et al., 2003; Kubisch et al., 2003; Muller et al., 2003). Here we characterize the distribution and function of the $\mathrm{CHC} 22$ isoform of clathrin heavy chain in skeletal muscle where it is preferentially expressed (Long et al., 1996; Sirotkin et al., 1996).

Clathrin is an essential component of several intracellular membrane traffic pathways. Its self-assembling triskelion structure allows the formation of clathrin-coated vesicles 
(CCVs) that sort membrane-bound nutrients, hormones, and proteases during receptor-mediated endocytosis and organelle biogenesis (reviewed in Brodsky et al., 2001). This familiar clathrin is formed by trimerization of the ubiquitous isoform of clathrin heavy chain (CHC17) that is encoded on human chromosome 17. Each $\mathrm{CHC} 17$ subunit is bound by a regulatory light chain (LC) of which there are two forms in vertebrates ( $\mathrm{LCa}$ and $\mathrm{LCb}$ ). The LCs control CHC17 assembly in the cell and interact with a number of accessory proteins involved in CCV function. The second isoform of clathrin heavy chain, $\mathrm{CHC} 22$, was discovered during the human genome project analysis of chromosome 22 (Kedra et al., 1996; Long et al., 1996; Sirotkin et al., 1996; Holmes et al., 1997). CHC22 mRNA was observed at high levels in skeletal muscle, somewhat lower levels in cardiac muscle and testes, and only at low levels in other tissues. The protein sequence of $\mathrm{CHC} 22$ predicts $85 \%$ identity with $\mathrm{CHC} 17$ protein, suggesting that they might have similar structural and functional properties. However, initial studies of $\mathrm{CHC} 22$ indicated that the two CHCs have different intracellular distributions, some distinct biochemical properties and are apparently regulated differently (Liu et al., 2001). These observations were primarily based on the properties of $\mathrm{CHC} 22$ expressed in vitro or expressed by transfection of HeLa cells or analysis of the low levels of endogenous CHC22 in HeLa cells. CHC22 was found to trimerize but it did not bind clathrin LCs in vitro, nor colocalize with LCs in cells. Nor did CHC22 Hub domain fragments (residues 1074-1640) alter LC distribution as seen following expression of the CHC17 Hub. In HeLa cells, CHC22 partially colocalized with the membrane adaptors AP1 and AP3 and could be immunoprecipitated with them, but showed no interaction with AP2. All three AP complexes are involved in linking CHC17 to membranes. Furthermore, CHC22 interaction with intracellular membranes was sensitive to exposure of cells to $20^{\circ} \mathrm{C}$ or cytochalasin $\mathrm{D}$, treatments that do not perturb CHC17 membrane binding. Distinct function and regulation of the two clathrin isoforms were further indicated by limited analysis of human skeletal muscle and myoblast cultures. Both clathrins were expressed in the former and staining revealed nonoverlapping distributions. $\mathrm{CHC} 22$ expression was minimal in undifferentiated myoblasts and increased during myogenesis in culture, whereas $\mathrm{CHC} 17$ expression was constant throughout.

There are multiple specialized aspects of muscle membrane traffic in which CHC22 could play a role. These include pathways that create and maintain muscle-specific membrane compartments such as T-tubules and sarcoplasmic reticulum or pathways that create and maintain domains of the sarcolemma that have organized contact with extracellular matrix (ECM) at the neuromuscular junction (NMJ), myotendinous junction (MTJ), and costameres. Defects in proteins involved in these matrix contact sites are associated with a number of hereditary muscle diseases (Clark et al., 2002; Ervasti, 2003). CHC22 could also potentially function in the specialized organization of housekeeping compartments. The Golgi apparatus and endocytic pathway components have characteristic locations in myofibers (Kaisto et al., 1999; Ralston et al., 1999), as do the more specialized membrane traffic compartments such as those that sequester the glucose transporter GLUT4 (Simpson et al., 2001).

Muscle membranes must be organized during myogenesis and repair, processes that also rely on specialized membrane fusion pathways. In the studies reported here, we characterize the localization of $\mathrm{CHC} 22$ in mature skeletal muscle and analyze its distribution during myogenesis and repair to establish its relationship with muscle membrane organization. Our findings are consistent with a role for $\mathrm{CHC} 22$ at sites of membrane contact with the ECM. We also observed a dramatic increase of $\mathrm{CHC} 22$ expression during muscle regeneration, indicating a role in muscle repair. These properties of $\mathrm{CHC} 22$ were distinct from the behavior and distribution of $\mathrm{CHC17}$, so we searched for $\mathrm{CHC} 22-$ binding proteins that did not bind $\mathrm{CHC17}$. One such protein identified was sorting nexin 5 (SNX5), which is highly expressed in skeletal muscle (Otsuki et al., 1999). In humans there are 22 SNXs, and all are characterized by a phox homology (PX) domain that mediates lipid binding and potential membrane association (Teasdale et al., 2001; Xu et al., 2001; Cozier et al., 2002). SNX proteins have been implicated in membrane cargo recognition and regulation of membrane traffic pathways (for a review see Worby and Dixon, 2002). We propose that the interaction of CHC22 with SNX5 explains some of the functional differences between $\mathrm{CHC} 22$ and $\mathrm{CHC} 17$, contributing to the specialization of $\mathrm{CHC} 22$ in muscle membrane pathways.

\section{MATERIALS AND METHODS}

\section{Tissue Collection, Preparation, and Immunofluorescence}

We obtained paraffin-embedded 5 - $\mu$ m-thick tissue sections of human skeletal muscle from postmortem cadavers from the Department of Pathology, UCSF (Figures 1, A-C and G-L, and 2, A-C). The paraffin was removed by submerging the samples twice, for $10 \mathrm{~min}$ each, in xylene in a glass slide holder in a fume hood. Samples were rehydrated in washes of $100 \%$ ethanol for $3 \mathrm{~min}$ and $70 \%$ ethanol for $3 \mathrm{~min}$ and $1 \mathrm{~min}$ in water. Before immunostaining, sections were treated with $0.05 \%$ saponin at room temperature for $30 \mathrm{~min}$ to unmask antigenic sites followed by three rinses in PBS. Blocking in a solution of $0.02 \%$ SDS, $0.1 \%$ NP- $40,1 \%$ cold water fish skin gelatin (FSG), and $1 \%$ BSA in PBS was carried out for $1 \mathrm{~h}$ at room temperature. Samples were incubated with primary antibody for $3 \mathrm{~h}$ in blocking solution at $37^{\circ} \mathrm{C}$ under a coverslip, followed by three 5-min-long washes at room temperature in blocking solution. The secondary antibody incubation was for $1 \mathrm{~h}$ in blocking solution at $37^{\circ} \mathrm{C}$ under a coverslip. Samples were then washed three times for $5 \mathrm{~min}$ each in blocking solution on a rotating platform and rinsed twice in PBS and once in water before being mounted in Vectashield mounting medium with DAPI (Vector Laboratories, Burlingame, CA).

Rat skeletal muscle was harvested from newborn rats (Figures 1, D-F, and 2, G-I. Supplementary Data 1 and 3A-I) or from adult rats (Figure 2, J-O). The tissue was fixed overnight in $4 \%$ paraformaldehyde in PBS and then washed 3 times in PBS (10 min each). Next the specimens were allowed to sink in a $15 \%$ sucrose solution before being embedded in optimum-cutting-temperature compound and frozen over chilled 2-methylbutane in liquid nitrogen Samples were stored at $-80^{\circ} \mathrm{C}$ until sectioning. Sections, $10 \mu \mathrm{m}$ thick, were collected on a cryostat at $-23^{\circ} \mathrm{C}$ on superfrost/plus-coated slides (Fisher Scientific, Pittsburgh, PA) and stored at $-80^{\circ} \mathrm{C}$. Samples were warmed to room temperature for $15 \mathrm{~min}$ before blocking in 3\% bovine serum albumin/ PBS for $30 \mathrm{~min}$ at room temperature. Primary antibody incubations were carried out in 1\% BSA/PBS for $2 \mathrm{~h}$ at room temperature under a coverslip, followed by three 5-min washes in PBS on a rotating platform. Specimens were then incubated with secondary antibodies for $1 \mathrm{~h}$ at room temperature under a coverslip, followed by washing as before. Finally samples were rinsed in distilled $\mathrm{H}_{2} \mathrm{O}$ and mounted in Vectashield containing DAPI (Vector Laboratories).

Regenerating rat muscle was isolated on days $0,1,3,5,7,10,14$, and 21 after injection of the right tibialis anterior muscle of male Wistar rats with Taiwan cobra venom, cardiotoxin (see Figures 5 and 6). Samples were collected at a location near the injection site but where inflammatory infiltrate from mechanical damage as a result of the injection was not detected. Inflammation was assessed by H\&E staining. Muscle biopsies were frozen in chilled isopentane cooled by liquid nitrogen and embedded. Serial cryosections were made for a previous study of regenerating muscle (Hoshino et al., 2001, 2002a $2003 b, 2002 c$ ) and were maintained frozen until used for this study. Immunofluorescence was carried out as described above for cryosections taken from normal rat muscle.

Human muscle cryosections (Figure 2, D-F) were obtained from the lab of Dr. Kimberly Topp (UCSF). Biopsies were taken from the vastus lateralis, frozen immediately in isopentane, cooled with liquid nitrogen, and stored at $-70^{\circ} \mathrm{C}$ until being sectioned. Cryosections were $10-\mu \mathrm{m}$-thick cross sections collected on Vecta-bond-treated slides (Vector Laboratories). Cryosections were made during the course of a study on the effects on human muscle after kidney transplantation (Topp et al., 2003). However, the sample shown in this study was from a healthy control patient. Slides were warmed to room 

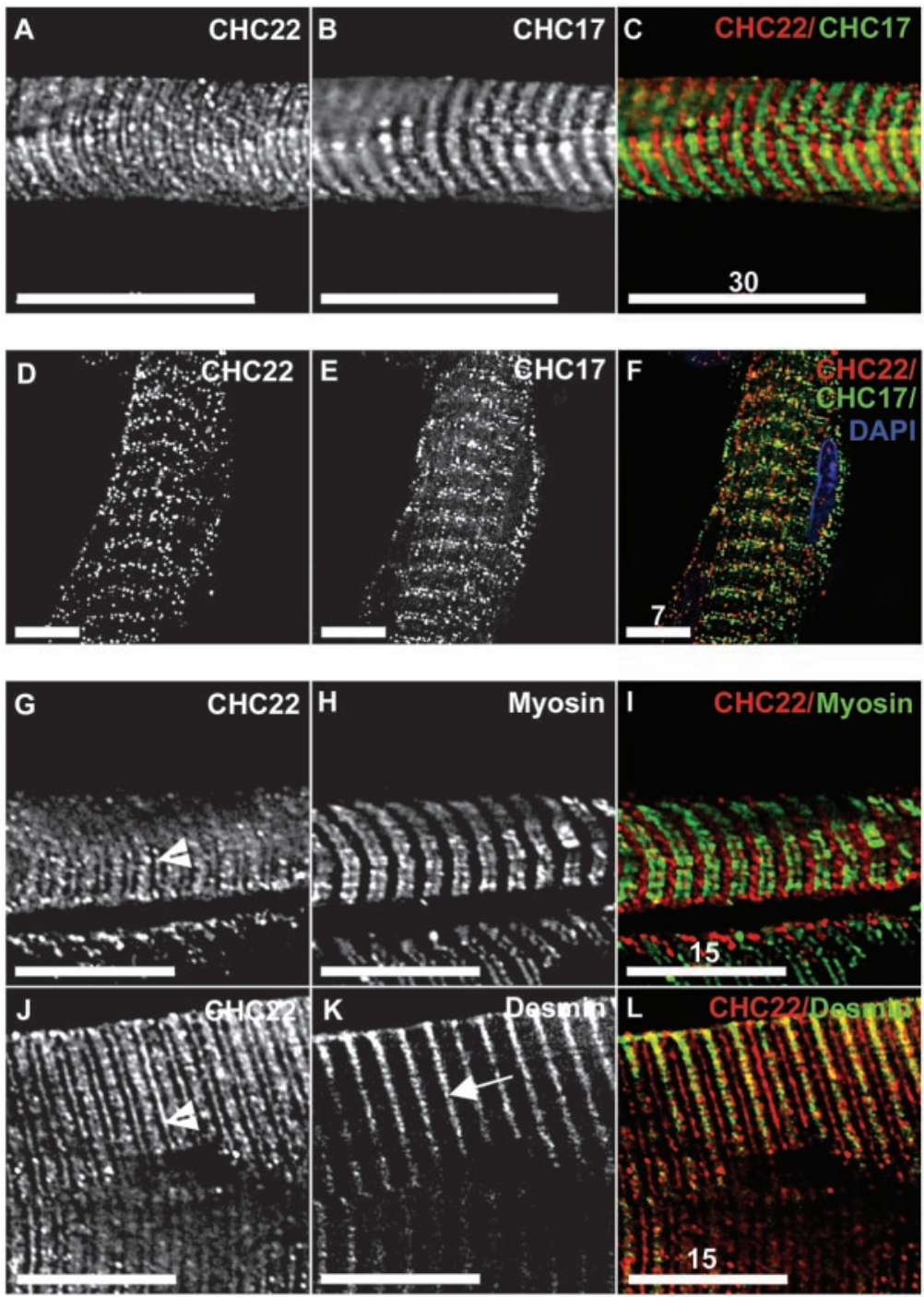

Figure 1. CHC22 distribution in human and rat skeletal muscle. Normal human muscle tissue was fixed, embedded in paraffin, and sectioned. Sections were prepared for immunofluorescence and labeled for $\mathrm{CHC} 22$ with a specific polyclonal antibody (A and C, red; G and $\mathrm{I}$, red; $\mathrm{J}$ and $\mathrm{L}$, red) and for $\mathrm{CHC} 17$ with $\mathrm{mAb}$ X22 (B and $\mathrm{C}$, green), monoclonal antibodies for myosin ( $\mathrm{H}$ and $\mathrm{I}$, green), and desmin ( $\mathrm{K}$ and $\mathrm{L}$, green). Arrowheads indicate the location of M-lines and the arrow indicates the location of a Z-disk. Normal rat muscle tissue was fixed and embedded in optimal cutting temperature compound for cryosectioning. Sections were prepared for immunofluorescence and labeled for $\mathrm{CHC} 22$ with a polyclonal antibody (D and $\mathrm{F}$, red) and for $\mathrm{CHC} 17$ with a mAb X22 (E and F, green). Nuclei were stained with DAPI (F, blue). Each row shows the same field viewed with different filters and the right hand panel shows the merged images. Scale bars in micrometers.

temperature for $15 \mathrm{~min}$ and then fixed lightly in $1 \%$ paraformaldehyde and washed three times for $5 \mathrm{~min}$ in PBS. Samples were processed for immunofluorescence as described for the cryosections above.

All rat tissue samples generated at UCSF were obtained through the committee of animal research tissue-sharing program.

\section{Cell Culture and Transfection}

Cell lines were grown at $37^{\circ} \mathrm{C}$ in a $5 \% \mathrm{CO}_{2}$ incubator. Rat myogenic cells, L8 were derived by David Yaffe from embryonic (or newborn) rat hind limbs (Yaffe and Saxel, 1977). The L8E63 cells are a clone derived from the L8 cell line in the laboratory of Stephen Kaufman (Kaufman et al., 1980). L8E63 cells were grown in DMEM supplemented with $10 \%$ horse serum, penicillin, streptomycin, and $2 \mathrm{mM}$ glutamine. They were passaged before reaching confluency to maintain a fusogenic population. To initiate the process of differentiation, cells were grown in low serum medium $(2 \%$ horse serum rather than 10\%; see Figure 4, A-D). However, L8E63 cells would fuse naturally if plated at 95-100\% confluency (Figure 4, H-M). For immunfluorescence studies cells were grown on collagen-coated cover slips to enhance their adherent qualities. Stable HeLa-tet/on cells (Clontech, Palo Alto, CA) containing the Tet response element, that express full-length or Hub $\mathrm{CHC} 22$ on addition of $2 \mu \mathrm{g} / \mu \mathrm{l}$ doxycycline, were maintained in DMEM supplemented with $10 \%$ Tet minus fetal bovine serum, penicillin, streptomycin, 2 $\mathrm{mM}$ glutamine, $0.4 \mathrm{mg} / \mathrm{ml}$ hygromycin B, and $0.2 \mathrm{mg} / \mathrm{ml}$ G418 (see Figure 7C; Liu et al., 2001). HeLa cells were cultured in DMEM (Life Technologies, Rockville, MD) supplemented with $10 \%$ fetal bovine serum, penicillin and streptomycin (Figure 7D). All transfections were carried out using Lipofectamine 2000 (Invitrogen, Carlsbad, CA) following the manufacturers instructions.

\section{Isolation and Immunofluorescence Analysis of Primary Rat Muscle Fibers and Primary Myoblasts}

Fibers were isolated from the musculus digitorum brevis muscle of adult rat and cultured on Matrigel-coated dishes in DMEM $+2 \%$ Serum Supreme + penicillin/streptomycin/L-glutamine (see Figures 3, J-L and 4, E-G). The fibers were fixed after overnight culture. Primary myotubes appeared after 5-7 days in culture in the presence of AraC to block cell division. The cells were washed with PBS and fixed with $4 \%$ PFA for $30 \mathrm{~min}$ at room temperature. After washing with PBS the cells were permeabilized with $0.5 \%$ TX-100 for $10 \mathrm{~min}$ at room temperature. Cells were then washed three times in PBS and nonspecific labeling was blocked for $30 \mathrm{~min}$ in $0.2 \%$ BSA $+0.2 \%$ FSG in PBS. Primary antibodies were diluted in blocking solution. Samples were incubated overnight at $4^{\circ} \mathrm{C}$. Samples were then washed with PBS three times for $5 \mathrm{~min}$ each. Secondary antibodies were diluted into the block solution and incubated at room temperature for $90 \mathrm{~min}$ (in the dark). After washing three times for 5 min each with PBS the samples were mounted with Mowiol. Samples were imaged by a Bio-Rad Radiance 2100 confocal microscopy system with LaserSharp 2000 software (Hercules, CA). A 100×, 1.3 oil immersion objective was used with a $2.5 \times$ digital zoom camera to collect the images. Images were further manipulated using Adobe Photoshop 5.0.

\section{Immunofluorescence Labeling of Cells}

Cells were rinsed twice in PBS, fixed in 4\% paraformaldehyde for $20 \mathrm{~min}$, and washed three times in PBS followed by incubation in $50 \mathrm{mM}$ ammonium chloride for $10 \mathrm{~min}$ and three washes in PBS. Permeablization was carried out using $0.2 \%$ (vol/vol) TX-100 for $4 \mathrm{~min}$. Again cells were washed three times in PBS, before blocking in $0.2 \%$ FSG (Sigma) in PBS for $10 \mathrm{~min}$. Antibodies were diluted in blocking solution and incubated with cells on cover slips for 

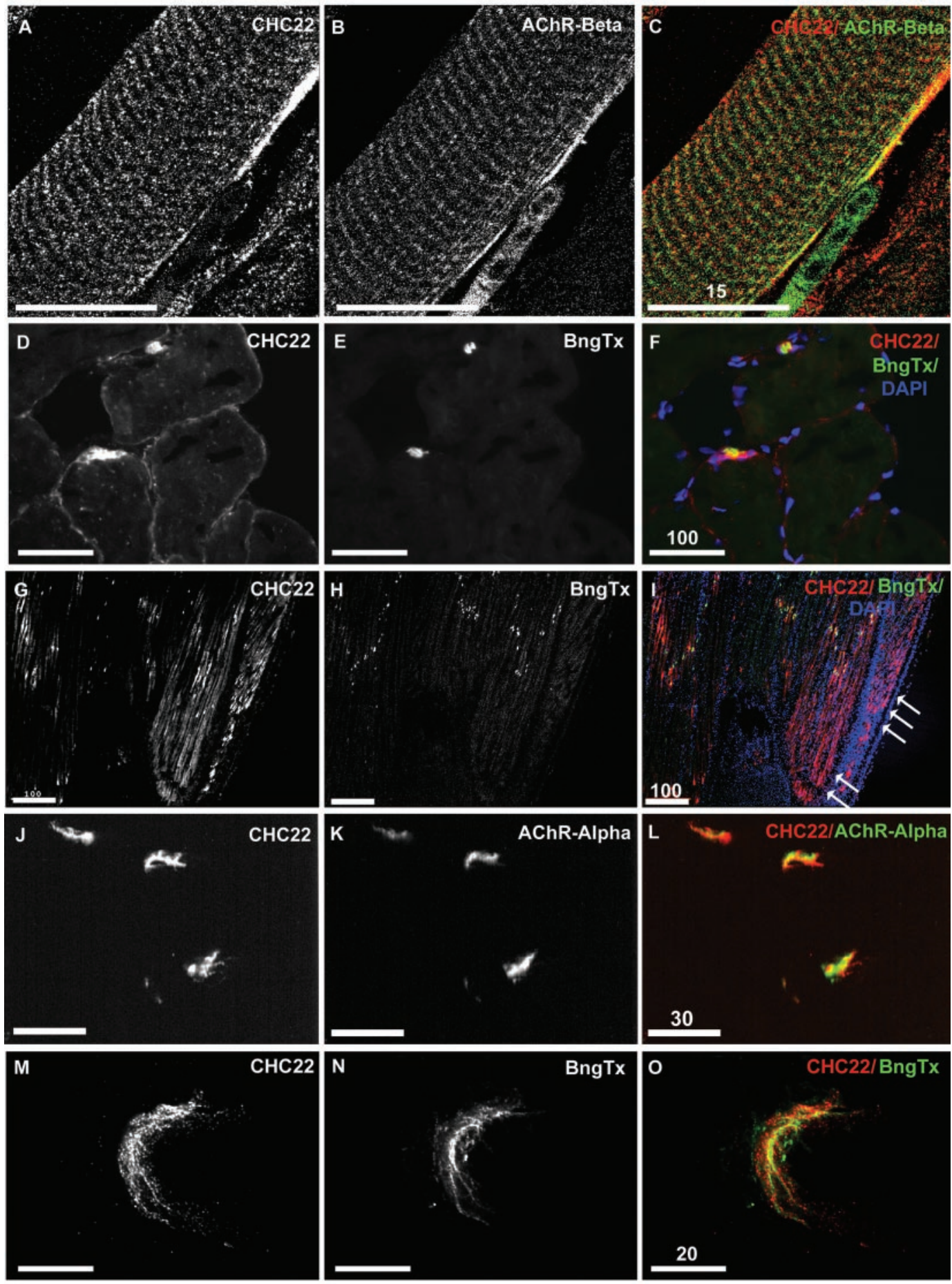

Figure 2. CHC22 concentrates at the neuromuscular junction of human and rat muscle. Normal human muscle tissue was sectioned and prepared for immunofluorescence and labeled for CHC22 with a polyclonal antibody (A and C, red; D and F, red) and for neuromuscular junctions using either antibody to acetylcholine receptor beta (AChR-Beta; B and C, green) or FITC-conjugated bungarotoxin (BngTx; E and F, green). Normal rat muscle tissue was fixed and embedded for cryo-sectioning. Sections were prepared for immunofluorescence and labeled for CHC22 with a polyclonal antibody ( $\mathrm{G}$ and I, red; J and L, red; $\mathrm{M}$ and $\mathrm{O}$, red) and for neuromuscular junctions (NMJs) using FITC-conjugated BngTx (H and I, green; $\mathrm{N}$ and $\mathrm{O}$, green) or antibody to acetylcholine receptor alpha (K and L, green). Arrows highlight where muscle contacts tendon (I). DAPI was used to stain nuclei (F and I, blue). Each row shows the same field viewed with different filters and the right-hand panel shows the merged images. Scale bars in micrometers.

$20 \mathrm{~min}$. Between primary and secondary antibody incubations cells were washed three times with PBS $/ 0.2 \%$ FSG. After incubations, cells were washed three times in PBS/0.2\% FSG, twice in PBS and once in water. Cover slips were mounted in Vectashield containing DAPI (Vector Laboratories). All steps were performed at room temperature (Figure 4, A-D and K-M, Supplementary Data 2). 


\section{Fluorescence Microscopy}

All images were acquired on a Deltavision Restoration microscope (Applied Precision Instruments, Issaquah, WA) using a MicroMax $5 \mathrm{MHz}$ cooled CCD camera (Roper Scientific, Tucson, AZ) and running SoftWoRx (Applied Precision) deconvolution and data analysis software.

\section{cDNA Constructs}

Full-length CHC22 cDNA was cloned into the HindIII-SmaI restriction sites of vector pEGFP-N3 from Clontech, for expression in L8E63 cells (see Figure 4, $\mathrm{H}-\mathrm{J}$ ). Full-length human SNX5 cDNA was cloned into the vector pCMU 3XFLAG conferring three FLAG epitope tags at the N-terminus (see Figure 7, $\mathrm{C}$ and D). For the mapping of SNX5/CHC22 interaction sites, clathrin heavychain fragments were amplified from human cDNAs by PCR and cloned into the EcoRI-SalI restriction sites of the pGBT9 bait vector (Clontech). SNX5 and clathrin light-chain $b$ fragments were amplified by PCR from the cDNA clones identified in the library screen and cloned into the EcoRI-XhoI restriction sites of the pACT2 prey vector (Clontech; see Figure 7, A and B).

\section{Yeast Two-hybrid Screening}

Two-hybrid screening was performed in the AH109 yeast strain. A human skeletal muscle library with cDNAs cloned into the prey vector pACT2 (Clontech) was screened using the hub of CHC22 as bait in the vector pGBT9. Yeast transformation and plate growth assays were performed following the Clontech yeast protocols handbook. One hundred clones were screened. Once positive clones were identified, plasmid DNA was isolated and transformed into KC8 E. coli cells. DNA was purified from KC8 cells and sequenced. Interactions were confirmed by cotransformation of yeast with the positive clones (SNX5 238-404 and clathrin light-chain b 59-212) and CHC22 hub. The minimal binding site of each partner was then determined by truncating each fragment and cloning them into the respective bait or prey vectors, followed by cotransformation and plate growth assay. Control fragments of $\mathrm{CHC} 17$ in bait vector pGBT9 were generated previously in our lab (Chen et al., 2002). For each indicated two-hybrid interaction, the cotransformation was repeated three times, each time with at least three colonies being picked and assayed (Figure 7B).

\section{Coimmunoprecipitation Studies}

HeLa cells that were permanently transfected to express full-length CHC22 (HeLa-Tet on-CHC22) or CHC22 Hub fragment (1074-1640; HeLa-Tet on$\mathrm{CHC} 22 \mathrm{Hub}$ ) under the inducible tetracycline promoter were found to constitutively express detectable levels of the transfected proteins. These cells were transiently transfected with SNX5, tagged at the N-terminus with the FLAG epitope (SNX5-FLAG). Cell lysates were prepared $16 \mathrm{~h}$ after transfection using buffer $(0.3 \mathrm{ml} / 100-\mathrm{mm}$ dish) containing $100 \mathrm{mM} \mathrm{NaCl}, 50 \mathrm{mM}$ HEPES, $\mathrm{pH} 7.4,5 \mathrm{mM} \mathrm{MgCl}, 0.5 \%$ NP-40, $1 \mathrm{mM}$ PMSF, and 1 protease inhibitor cocktail tablet $(1 / 10 \mathrm{ml}$ buffer, Roche, Indianapolis, IN). Lysates were centrifuged at $14,000 \mathrm{rpm}$ at $4^{\circ} \mathrm{C}$ for $10 \mathrm{~min}$, and the supernatants were kept for immunoprecipitations. For Figure $7 \mathrm{C}$, lysates were incubated with anti-CHC22 antibody for $2 \mathrm{~h}$ on a rotating wheel at $4^{\circ} \mathrm{C}$, protein G-Sepharose (PGS) 4 fast flow (Amersham Biosciences, Piscataway, NJ) was added for $1 \mathrm{~h}$ and beads were washed three times in lysis buffer containing 1\% TX-100 instead of NP-40. Samples were resolved by SDS-PAGE on $4-12 \%$ gradient NuPAGE gels, using MOPS running buffer (Invitrogen). Proteins were transferred onto nitrocellulose membrane by Western blot and detected by antibody labeling and chemiluminescence. For Figure 7D, immunoisolations were carried out from HeLa cell lysates using polyclonal antibodies specific for $\mathrm{CHC} 22$ or $\mathrm{CHC} 17$ ( $\alpha$-cons). After immunoprecipitating the clathrin heavy chains, PGS beads were washed twice in lysis buffer and then incubated for $1 \mathrm{~h}$ with lysate of SNX5-FLAG-transfected HeLa cells or mock-transfected HeLa cells. PGS beads were washed three times in lysis buffer. Samples were resolved by SDS-PAGE on $4-12 \%$ gradient NuPAGE gels, using MOPS running buffer (Invitrogen). Proteins were transferred onto nitrocellulose membrane by Western blot and detected by antibody labeling and chemiluminescence.

\section{Antibodies}

The antibodies used in this study were as follows: rabbit polyclonal and monoclonal anti-CHC22 antibodies generated in our lab to the trimerization domain of CHC22 (1521-1640) as described in Liu et al. (2001). Monoclonal antibodies to CHC17 (TD.1 and X22) and to LCa (X16) and LCb (LCB.1) and polyclonal antibodies against clathrin light chains ( $\alpha$-cons) were generated in the Brodsky lab (Blank and Brodsky, 1986; Acton and Brodsky, 1990; Nathke et al., 1990). Antibodies to Ankrd2, FATZ, ZASP, and Telethonin were from Georgine Faulkner, International Centre of Genetic Engineering and Biotechnology, Italy. Antibody to GLUT4 was from Jeffrey Pessin, University of Michigan, Ann Arbor. Sources of antibodies recognizing the following adaptor proteins were AP1 (100.3) and AP2 (100.2) from Sigma, AP3 from Regis Kelly, University of California, San Francisco, and AP4 from Juan Bonifacino, $\mathrm{NIH}$. The polyclonal antibody to GGA1 was from Margaret Robinson, University of Cambridge, England. The mouse mAb to synaptic vesicle protein 2 and ascites mouse monoclonal against $\alpha 7$ Integrin (H36) were from Robert
Edwards, University of California, San Francisco and Stephen Kaufman, University of Illinois, Urbana, Illinois, respectively. Purchased antibodies were as follows: monoclonal against myosin heavy chain of fast skeletal muscle (MY-32), monoclonal against actin clone AC-40, monoclonal against sarcomeric actin and anti-FLAG M2 mAb (Sigma), mouse monoclonal to $\mathrm{M}$-cadherin and rabbit polyclonal caveolin 3 (BD Transduction Laboratories, Lexington, KY), mouse monoclonals to nestin, acetylcholine receptors alpha/ beta, integrin alpha 1 and dihydropyridine receptor (Chemicon International, Temecula, CA), fluorescein conjugated $\alpha$-bungarotoxin (Molecular Probes, Eugene, OR), mouse monoclonal to ryanodine receptor (Biomol), mouse monoclonal to troponin I and dystrophin (AbCam, Cambridge, MA), mouse monoclonal to TGN38 (Transduction Labs) and mouse to desmin (Zymed Labs, South San Francisco, CA). Mouse monoclonal MyHC against embryonic myosin (F1.652) was obtained from the Developmental Studies Hybridoma Bank, University of Iowa. Secondary antibodies purchased from Jackson ImmunoResearch Laboratories (West Grove, PA) were fluorescein (FITC)conjugated AffiniPure donkey anti-mouse IgG, rhodamine red-X-conjugated AffiniPure donkey anti-rabbit IgG, and Cy-5-conjugated AffiniPure donkey anti-mouse IgG; those from Molecular Probes were donkey anti-mouse Alexa 488 and anti-rabbit Alexa 546.

\section{RESULTS}

\section{CHC22 Concentrates at Neuromuscular and Myotendinous Junctions}

Antibodies that distinguish between human $\mathrm{CHC} 22$ and CHC17 (Liu et al., 2001) were used to stain sections of mature human and rat skeletal muscle. The differential distribution of the two clathrins was confirmed in both species (Figure 1, A-F) and both displayed a striated pattern of punctate staining, typical of intracellular vesicles. The distribution of $\mathrm{CHC} 22$ relative to a number of muscle structural proteins and proteins associated with muscle membranes was then characterized in mature human skeletal muscle (Table 1, Figures 1 and 2). Consistent with a lack of CHC17 colocalization, $\mathrm{CHC} 22$ staining did not overlap with staining for clathrin LCs. In contrast to what was previously observed in HeLa cells, staining of human skeletal muscle showed no overlap between $\mathrm{CHC} 22$ staining patterns and AP1, AP2, or AP3 adaptors. This observation suggests that in muscle tissue, where the expression of CHC22 is highest, it interacts with tissue-specific proteins that occupy adaptor binding sites and are most likely not expressed in HeLa cells. There was also no colocalization of CHC22 with GLUT4 or of CHC22 with T-tubule markers dihydropyridine receptor, ryanodine receptor or caveolin 3 (Table 1). In longitudinal sections of human muscle, the only structural protein that showed partial colocalization with $\mathrm{CHC} 22$ was desmin. Staining patterns of the two proteins overlapped at the periphery of Z-disks (Figure 1, J-L). CHC22 did not colocalize with myosin (Figure 1, G-I), actin, any Z disk components analyzed or myofibrillar components (Table 1). CHC22 appeared to localize to the same region as M-lines (Figure 1, G and J, arrowheads), but the staining pattern was consistent with vesicular staining in the region, rather than characteristic of an M-line structural protein. CHC22 staining partially overlapped with labeling for dystrophin at the sarcolemma (Table 1) and for acetylcholine receptors labeled either by antibodies (Figure 2, A-C) or by bungarotoxin (BngTx) binding (Figure 2, D-F). In transverse sections of human skeletal muscle, it was apparent that $\mathrm{CHC} 22$ and acetylcholine receptors were both concentrated at NMJs, although $\mathrm{CHC} 22$ antibody labeling did not completely COincide with bungarotoxin (BngTx) binding to NMJs (Figure 2, D-F).

Localization of CHC22 to NMJs was confirmed in both longitudinal and transverse sections of rat skeletal muscle (Figure 2, G-O), where it also concentrated at typically crescent-shaped structures labeled for acetylcholine receptors, but without complete overlap with receptor labeling. In rat 
Table 1. Proteins analyzed for distribution relative to $\mathrm{CHC}_{2}{ }^{\mathrm{a}}$

\begin{tabular}{|c|c|c|}
\hline & Human skeletal muscle & L8 cells \\
\hline $\begin{array}{l}\text { Membrane transport } \\
\text { markers }\end{array}$ & $\begin{array}{l}\text { CHC17 } \\
\text { Clathrin light chain } \mathrm{a}+\mathrm{b} \\
\text { AP1 } \\
\text { AP2 } \\
\text { AP3 } \\
\text { GLUT4 }\end{array}$ & $\begin{array}{l}\text { CHC17 } \\
\text { Clathrin light chain } \mathrm{a}+\mathrm{b} \\
\text { AP2 } \\
\text { AP3 } \\
\text { AP4 } \\
\text { GGA1 } \\
\text { TGN38 }\end{array}$ \\
\hline $\begin{array}{l}\text { Skeletal muscle } \\
\text { markers }\end{array}$ & $\begin{array}{l}\text { Caveolin } 3 \\
\text { Dihydropyridine receptor } \\
\text { Ryanodine receptor } \\
\text { Myosin } \\
\text { Actin } \\
\text { Alpha-actinin } \\
\text { Sarcomeric actin } \\
\text { Desmin } \\
\text { Dystrophin } \\
\text { AChR alpha/beta } \\
\text { Integrin alpha } 1 \\
\text { ZASP } \\
\text { Telethonin }\end{array}$ & $\begin{array}{l}\text { Caveolin } 3 \\
\text { Dihydropyridine receptor } \\
\text { Ryanodine receptor } \\
\text { Myosin (fast) } \\
\text { Troponin I } \\
\text { Alpha-tubulin } \\
\text { Desmin } \\
\text { Nestin } \\
\text { Dystrophin } \\
\text { Integrin alpha } 7^{\mathrm{b}} \\
\text { M-Cadherin } \\
\text { Alpha-actinin } \\
\text { Telethonin } \\
\text { Ankrd2 } \\
\text { ZASP } \\
\text { FATZ }\end{array}$ \\
\hline
\end{tabular}

${ }^{a}$ Human skeletal muscle sections or L8E63 cells were labeled with monoclonal or polyclonal antibodies against the proteins listed and the staining patterns compared to labeling with polyclonal anti-CHC22. In cases where double labeling was possible, comparison was made on the same tissue section. All the antibody staining patterns were different from the CHC22 pattern. Those antibodies whose staining pattern partially overlapped with CHC22 (in double label experiments) are indicated by footnote. L8E63 cells were examined for CHC22 colocalization with the proteins indicated on several sequential days after serum withdrawal. AChR, acetylcholine receptor; ZASP, z-disk associated protein; Ankrd2, ankyrin rich protein; FATZ, a filamin-, actinin-, and telethonin-binding protein of the Z-disc (expressed in the same cells as CHC22 but no overlap); GLUT4, glucose transporter 4.

bPartial overlap.

skeletal muscle sections, CHC22 distribution at the NMJ was further characterized relative to $\mathrm{CHC} 17$, to the neuronal synaptic vesicle 2 (SV2) protein and to nestin, an intermediate filament protein specific for NMJs and MTJs (Supplementary Data 1; Carlsson et al., 1999; Vaittinen et al., 1999). CHC22 was clearly on the opposite side of the NMJ to SV2 and was visible in cross-striations on the postsynaptic side. CHC17 was not concentrated at NMJs as observed for $\mathrm{CHC} 22$. Although $\mathrm{CHC} 22$ localized to the same NMJ structures as nestin, there was only partial colocalization of staining for the two proteins. In the longitudinal section of rat muscle (Figure 2, G-I), the zigzag distribution of BngTx labeling, characteristic of innervation patterns, was mirrored by CHC22 labeling, again without staining being fully coincident.

Also evident in the same section was a concentration of $\mathrm{CHC} 22$ at the MTJ (arrows in Figure 2I). Specific localization of $\mathrm{CHC} 22$ to the MTJ was further established by comparing its distribution to $\mathrm{CHC} 17$, nestin and $\alpha 7$ integrin in this region of rat skeletal muscle (Figure 3). The $\alpha 7$ integrin is localized to sites of muscle contacts with ECM at both the NMJ and MTJ (Burkin and Kaufman, 1999). As seen at the NMJ, CHC17 distribution at the MTJ was uniform compared with $\mathrm{CHC} 22$ and $\mathrm{CHC17}$ was more evenly dispersed in internal striations of skeletal muscle in the MTJ region (Figure $3, \mathrm{~A}-\mathrm{C})$. CHC22 and nestin or $\alpha 7$ integrin were concentrated at the MTJ, but the staining patterns were not completely coincident (Figure 3, D-I). Notable in these images is the density of nuclei characteristic of the mononuclear tendon tissue that is contacted by muscle fibers at the MTJ.
CHC22 staining of isolated rat myofibers was also observed to be concentrated at the MTJ, where it partially localized with desmin, which interacts with nestin at the MTJ (Figure $3, \mathrm{~J}-\mathrm{L})$. In summary, analysis of human and rat skeletal muscle sections with an antibody to human $\mathrm{CHC} 22$ revealed that, in skeletal muscle from both species, CHC22 is present in internal vesicles organized in a striated pattern. Also, in both human and rat skeletal muscle, $\mathrm{CHC} 22$ is concentrated at NMJs and MTJs and has a distinct distribution from $\mathrm{CHC} 17$ clathrin, which is not preferentially concentrated at these sites.

\section{Polarized Distribution of CHC22 during Myogenesis}

We have previously shown that $\mathrm{CHC} 22$ protein expression is increased $\sim 50$-fold in differentiating human myoblasts (SKMC cells), whereas CHC17 expression remains constant (Liu et al., 2001). To study this process morphologically, $\mathrm{CHC} 22$ expression was characterized in the more robust myogenic rat cell line, L8E63 (Figure 4 and Supplementary Data 2). These cells were induced to differentiate by withdrawal of serum from the culture medium and then doublelabeled with antibodies recognizing CHC22 and CHC17. $\mathrm{CHC} 22$ was undetectable in the undifferentiated cells (Figure $4 \mathrm{~A})$. By day 2, CHC22 was visible and its distribution pattern was clearly distinct from that of $\mathrm{CHC} 17$ (Figure 4B). CHC22 localized to the poles of multinuclear myosacs formed by these cells, whereas $\mathrm{CHC} 17$ was distributed throughout the multinuclear cells and showed its characteristic concentration at the trans-Golgi network (Figure 4, C and D). CHC22 localization was also analyzed in a culture of 

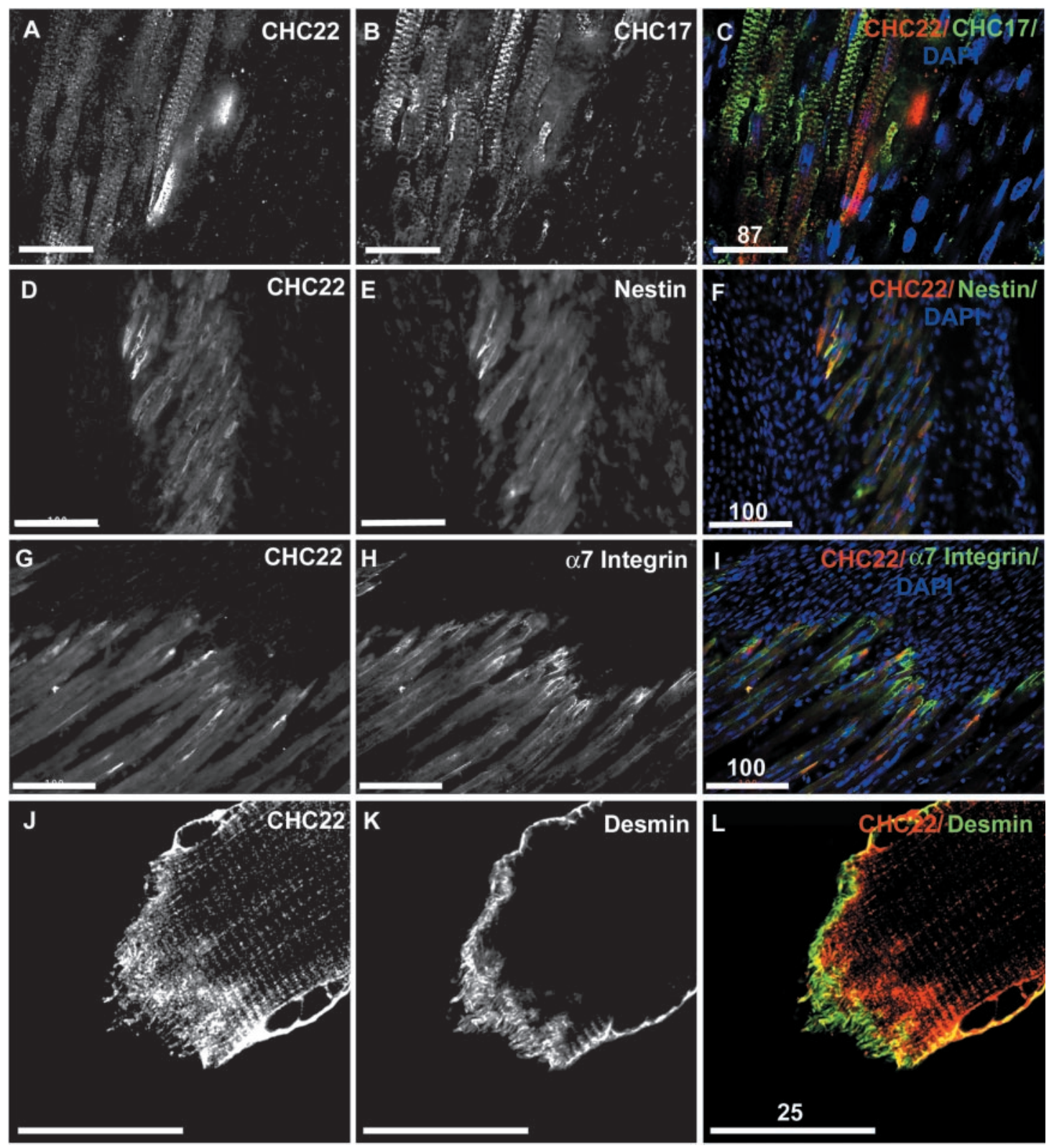

Figure 3. $\mathrm{CHC} 22$ concentrates at myotendinous junctions. Rat muscle tissue was fixed and embedded for cryosectioning. Sections were prepared for immunofluorescence and samples were double-labeled with antibodies to CHC22 (A and C, red; D and F, red; G and I, red), and antibodies to CHC17 (B and C, green), nestin (E and F, green), and $\alpha 7$ integrin (H and I, green). Nuclei are stained with DAPI (C, F, and I, blue). Regions where there are many nuclei but no staining with the other markers are tendons. Isolated myofibers were harvested from rat skeletal muscle (musculus digitorum brevis) and cultured on matrigel. The fiber shown was fixed and labeled for CHC22 (J and L, red) and desmin (K and L, green). Each myofiber has one NMJ and one MTJ. The MTJ region is shown. Each row shows the same field viewed with different filters and the right-hand panel shows the merged images. Scale bars in micrometers.

primary rat myoblasts isolated from rat foot skeletal muscle (Figure 4, E-G). Myoblasts were induced to fuse by serum reduction (in the presence of mitotic inhibitor AraC) and then double-labeled for immunofluorescent staining with antibodies to $\mathrm{CHC} 22$ or caveolin 3 (muscle-specific isoform of caveolin). CHC22 localized to the poles of fused myoblasts (Figure 4, F and G), as seen in differentiating L8E63 cells (Figure 4, C and D), and its distribution was distinct from that of caveolin 3 (Figure 4, E and G). The expression levels of $\mathrm{CHC} 22$ detected during differentiation of L8E63 cells correlated with that expected from expression studies of human $\mathrm{CHC} 22$ over the same differentiation time course for the human myoblast cell line SKMC. However, the antihuman $\mathrm{CHC} 22$ antibody used for these experiments, which immunoprecipitates CHC22 from SKMC cells, was not able to immunoprecipitate $\mathrm{CHC} 22$ from differentiated L8E63 cell lysate, likely because of reduction in affinity for the rat orthologue. The comparable immunofluorescence patterns in Figures 1 and 2 indicate that the antibody used recognizes the orthologous $\mathrm{CHC} 22$ protein in rat and human skeletal muscle sections and does not recognize CHC17. The antibody did not recognize CHC17 in the L8E63 cells (Figures 4 , A-D). To confirm that it recognized the rat orthologue of human $\mathrm{CHC} 22$, the anti-human $\mathrm{CHC} 22$ antibody staining of 


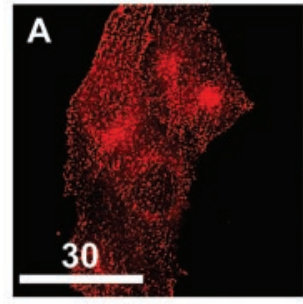

Day 0

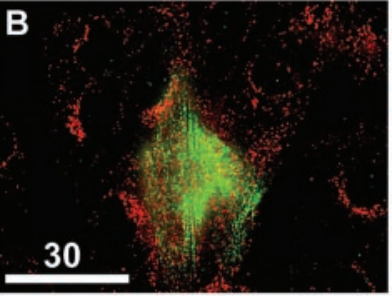

Day 2

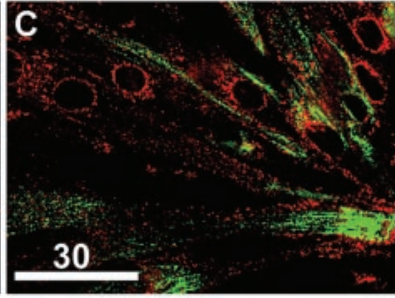

Day 4

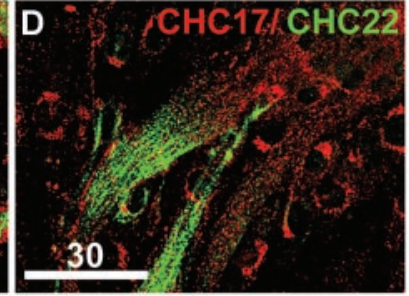

Day 6
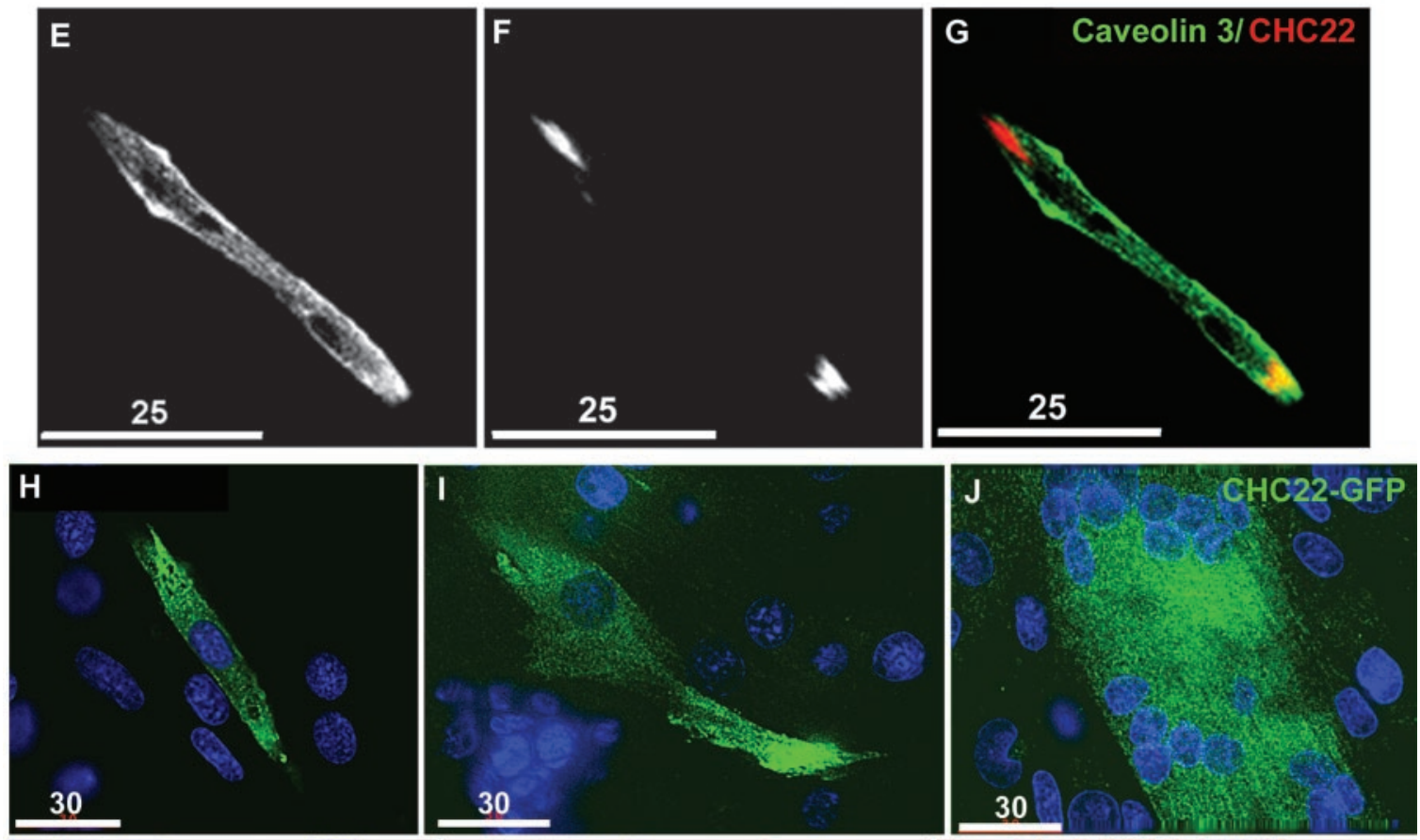

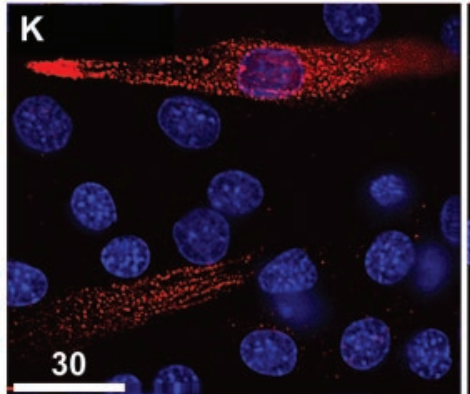

Day 1

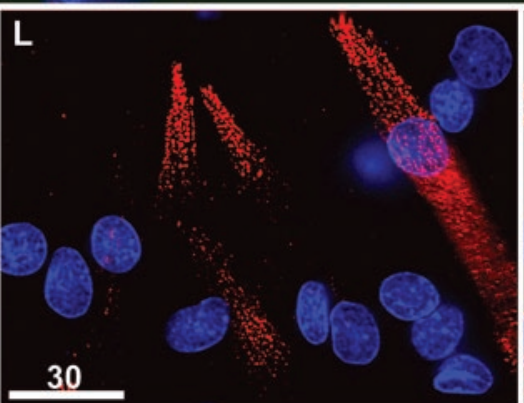

Day 3

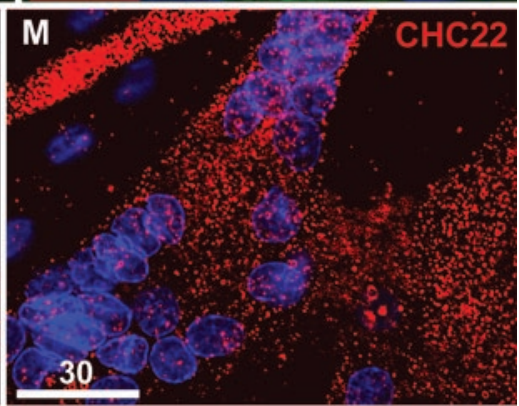

Day 7

Figure 4. CHC22 expression increases during myogenesis and transiently acquires a polarized distribution. Rat L8E63 skeletal muscle cells grown on collagen-coated cover slips were induced to differentiate by serum starvation at day 0 . Cells were fixed on the subsequent days indicated and processed for immunofluorescence, staining for $\mathrm{CHC} 22$ using a polyclonal antibody (A-D, green) and for $\mathrm{CHC} 17 \mathrm{using} \mathrm{mAb}$ X22 (A-D, red). Rat primary skeletal myoblasts grew out of the cultures described in the legend to Figure 3 (J-L). Shown are two cells that appear to have recently fused to initiate myotube formation. Cells were fixed and labeled for caveolin 3 (E and G, green) and CHC22 (F and $\mathrm{G}$, red). The same field viewed with different filters and the right-hand panel shows the merged images (E-G). Rat L8E63 skeletal muscle cells grown on collagen-coated cover slips were induced to differentiate by serum starvation at day 0. Cultures were transfected with GFP-tagged full-length human CHC22 (CHC22-GFP) 24 h before fixation, and then they were fixed on day 1 (H), day 3 (I), and day 7 (J) after serum starvation and processed for immunofluorescence analysis. The transfected CHC22 was visualized by direct fluorescence (H- J, green). For comparison of parallel cultures, L8E63 cells were fixed on day $1(\mathrm{~K})$, day 3(L), and day $7(\mathrm{M})$ after serum starvation and labeled with polyclonal antibody against $\mathrm{CHC} 22(\mathrm{~K}-\mathrm{M}$, red). The degree of differentiation is evident from the formation of multinucleated myosacs, which can be seen by the nuclear DAPI staining $(\mathrm{H}-\mathrm{M}$, blue). Scale bars in micrometers.

L8E63 was compared with the distribution of CHC22 fused to green fluorescent protein (CHC22-GFP) transfected into these cells (Figure 4, H-M). It can be seen that the transfected CHC22-GFP protein (Figure 4, H-J) had the same distribu- tion as the anti-CHC22 staining of untransfected cultures differentiated at the same time (Figure 4, K-M). On days 1 and 3 of differentiation both patterns were polarized and both became more diffuse on day 7 of differentiation, indi- 
cating a peak of polarized $\mathrm{CHC} 22$ activity at intermediate stages of myogenesis. The polarized localization pattern for $\mathrm{CHC} 22$ during these intermediate stages suggests a role for the protein at sites where the growing multinuclear myoblast is attached to a substrate. This is consistent with CHC22 localization to the MTJ and NMJ in mature muscle, which are sites of muscle-ECM interaction. The comparable staining patterns between rat and human mature skeletal muscle and between the CHC22-GFP protein transfected into L8E63 cells and that recognized by the antibody, along with the identical time course of expression of the detected protein in differentiating rat and human myoblasts, as well as the lack of cross-reactivity of the anti-human $\mathrm{CHC} 22$ with either rat or human $\mathrm{CHC17}$, together indicate that the antihuman $\mathrm{CHC} 22$ is recognizing the rat orthologue.

A number of structural proteins that function in muscle differentiation and in mature muscle were tested for colocalization with $\mathrm{CHC} 22$ during L8E63 myogenesis (Table 1 and Supplementary Data 2). Most of the proteins tested had completely distinct staining patterns compared with $\mathrm{CHC} 22$ (Table 1 ), including T-tubule, myofibrillar, and $Z$ disk proteins as well as M-cadherin and dystrophin. Distribution of integrin $\alpha 7$ partially overlapped with $\mathrm{CHC} 22$ staining at myotube tips, as observed in mature skeletal muscle at MTJs. Two intermediate filament proteins (desmin and nestin) also showed partial colocalization with $\mathrm{CHC} 22$, restricted to early stages of differentiation (Supplementary Data 2). By day 4, CHC22 distribution was increasingly distinct from the two intermediate filament proteins, both of which were less concentrated at the poles of the multinuclear myosacs, relative to $\mathrm{CHC} 22$. Additionally, at day 4 , some cells expressed desmin and nestin but did not stain for $\mathrm{CHC} 22$, indicating heterogeneity in the induced differentiation of L8E63 cells. These staining patterns again suggest localization of $\mathrm{CHC} 22$ to muscle contacts with the ECM. Consistent with analysis of mature skeletal muscle, CHC22 staining patterns did not overlap with antibodies labeling proteins associated with $\mathrm{CHC} 17$-coated vesicles, including the $\mathrm{AP}$ and GGA1 adaptor molecules. There was also no coincidence with the vesicle traffic marker TGN38. The lack of colocalization with AP1 and AP3, which partially colocalized with CHC22 in HeLa cells, again suggests tissue-specific proteins bind CHC22 when myogenesis is initiated, displacing potential interactions with CHC17-binding proteins.

\section{CHC22 Expression Is Increased in Regenerating Muscle after Cardiotoxin Injection}

An increase of $\mathrm{CHC} 22$ expression during myogenesis indicated a role in muscle development, suggesting that $\mathrm{CHC} 22$ might also play a role in muscle repair. Tissue sections of biopsies obtained from regenerating rat skeletal muscle were therefore analyzed for CHC22 expression (Figure 5). These biopsies were sampled at defined intervals of recovery following cardiotoxin injection into rat tibialis anterior muscle. Serial sections of the same biopsies were previously analyzed for the expression patterns of a number of muscle structural proteins (Hoshino et al., 2001, 2002a, 2002b, 2002c), so these samples have already been established and characterized as models of muscle regeneration. For comparison to the regenerating muscle as a control, muscle was injected with saline, sampled in the same manner, and then analyzed for $\mathrm{CHC} 22$ expression. In transverse sections of control rat muscle, faint sarcolemmal staining for CHC22 was observed as well as concentrated staining at NMJs (Figure 5A). On day 1 after cardiotoxin injection, the tissue undergoes massive necrosis as seen by the disorganized morphology of the tissue (Figure 5B). CHC22 staining was also disorganized.
By day 3, satellite cells infiltrate the muscle and immature regenerating myofibers with centrally located nuclei appear (Figure 6). The intensity of CHC22 staining was increased at this time point, relative to the earlier days of regeneration (Figures 5C and 6, A, D, and G), and CHC22 staining was predominantly in the interior of regenerating myofibers with central nuclei (Figure 6, C, F, and I). On day 5 regenerating myofibers with small diameters make up the majority of the affected tissue, and here $\mathrm{CHC} 22$ was observed concentrated at the sarcolemmal perimeter of these myofibers (Figure 5D). Days 7 and 10 reflect the growth of myofibers into larger regenerating fibers and CHC22 staining became less uniform and more localized at the sarcolemma in regions where gaps between regenerating fibers were still observed (Figure 5, E and F). It has been established that reinnervation occurs around day 14 and at this time point as well as day 21, CHC22 staining was concentrated at NMJs, as seen in control tissue (Figure 5, A, G, and H), i.e., in crescent shapes, polarized to one side of the myofiber. CHC17 distribution was analyzed at all stages of regeneration and in control muscle, and its distribution was uniform compared with that of CHC22 (Figure 6, B and C, and unpublished data). There were no stages of differentiation in which CHC17 expression appeared to increase or in which its distribution was polarized. To further analyze the stage of regeneration in which $\mathrm{CHC} 22$ expression was dramatically increased, muscle samples from day 3 after cardiotoxin injection were stained for established markers of muscle regeneration, M-cadherin (Figure 6, E and F; Matsuda et al., 1983) and embryonic myosin (Figure 6, H and I; Matsuda et al., 1983; Karsch-Mizrachi et al., 1989). Eighty-four percent of fibers $(\mathrm{n}=100)$ that stained for embryonic myosin also expressed $\mathrm{CHC} 22$, and $50 \%$ of $\mathrm{CHC} 22$-positive fibers $(\mathrm{n}=$ 100) also expressed M-cadherin. Neither marker had a staining pattern that substantially coincided with $\mathrm{CHC} 22$ labeling, although present in the same fibers. CHC22 staining was also observed at the sarcolemma of large diameter myofibers that appeared unaffected by cardiotoxin treatment (Figure 6, G and I). The staining pattern for $\mathrm{CHC} 22$ in regenerating muscle was compatible with repair functions for $\mathrm{CHC} 22$ at two stages of muscle recovery. These include potential roles at the interface between myofibers and satellite cells where M-cadherin is observed and/or in reestablishing fiber-fiber or fiber-nerve contacts. Furthermore, the redistribution of $\mathrm{CHC} 22$ from uniform expression in regenerating fibers to concentrated sites at the NMJ was similar to that reported for other proteins, such as nestin, involved in NMJ organization and function, whose localization is dependent on signals that occur upon reinnervation (Vaittinen et al., 1999).

\section{CHC22 Interacts with Sorting Nexin 5}

The strikingly different distribution of CHC22 compared with that of $\mathrm{CHC} 17$ in myoblasts and mature muscle and the lack of colocalization of $\mathrm{CHC} 22$ with the regulatory clathrin light-chain (LC) subunits or with CCV adaptor proteins in muscle suggest there are specific binding partners for $\mathrm{CHC} 22$ that are expressed in muscle. To identify such binding partners, the CHC22 Hub fragment (1074-1640) was cloned into a bait vector for yeast two-hybrid screening and was tested for interactions with a prey library derived from human skeletal muscle. Candidate binding proteins identified were then tested by two-hybrid assay for lack of interaction with the CHC17 Hub fragment to determine which ones might be involved in regulating the differential behavior, distribution, and apparently different functions of the two clathrins. A fragment of sorting nexin 5 (residues 238- 

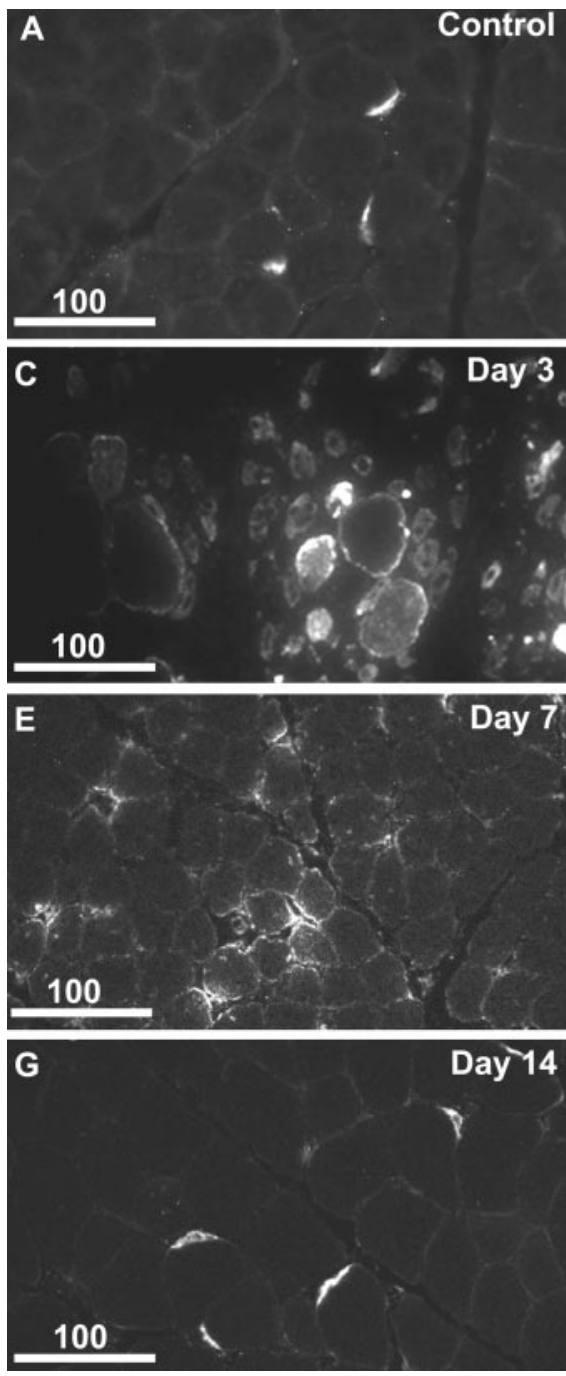
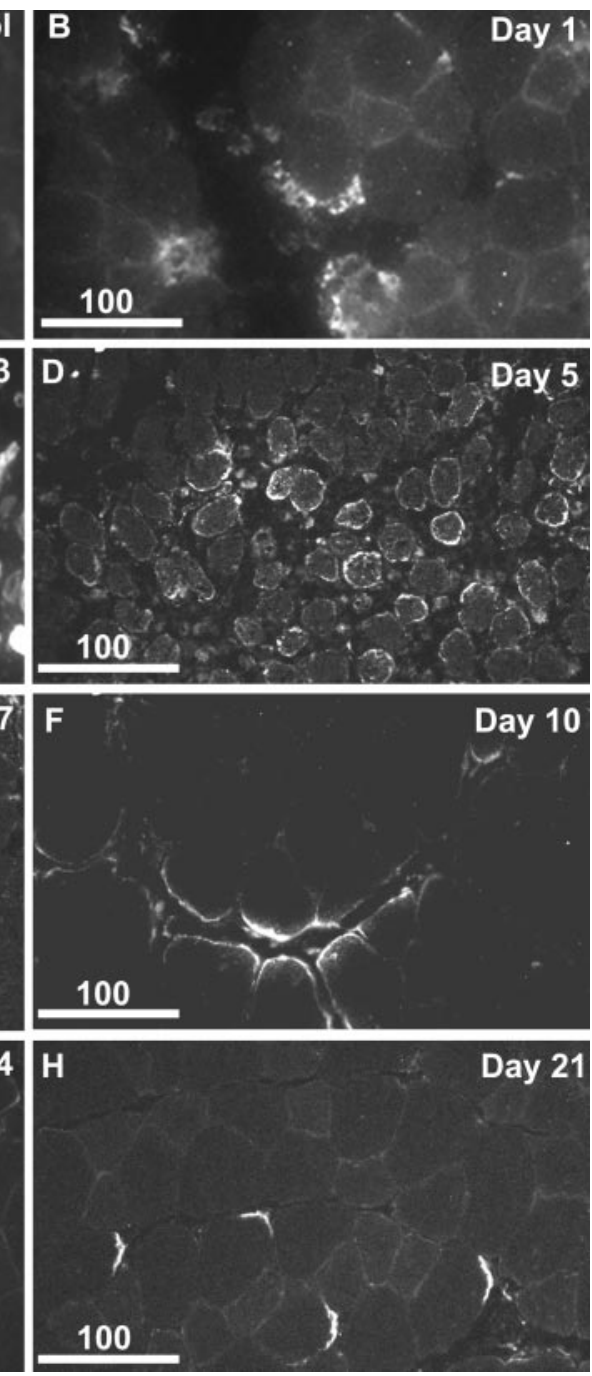

Figure 5. $\mathrm{CHC} 22$ expression is increased in regenerating rat muscle. Transverse section of rat tibialis anterior muscle collected after saline injection (A), as a control for injection of cardiotoxin venom. Transverse sections of rat tibialis anterior muscle collected on days 1,3 , $5,7,10,14$, and 21 after injection of cardiotoxin venom (B-H). Tissue was fixed and processed for immunofluorescence analysis of CHC22 distribution using polyclonal antiCHC22. Scale bars in micrometers.
404) was one of the prey constructs that interacted with CHC22 Hub but not with CHC17 Hub (Figure 7, A and B). Because sorting nexins are implicated in membrane binding, vesicle cargo binding and membrane traffic (Worby and Dixon, 2002), SNX5 was chosen for further analysis to confirm its status as a CHC22-binding protein. Furthermore, published tissue distribution data for SNX5 reported differential high expression in skeletal muscle with secondary expression in kidney, and expression in some transformed cell lines but very little in HeLa cells (Otsuki et al., 1999), supporting the possibility that SNX5 represents a functional CHC22-binding partner in muscle.

Sequence analysis on the National Center for Biotechnology Information Aceview website predicted that $\mathrm{CHC} 22$ has a coiled-coil 4 (CC4) domain at residues 1459-1488 within the Hub domain that is not predicted for CHC17. Two CC4 domains were already predicted for SNX5 at residues 259289 and residues 340-380 (Worby and Dixon, 2002). CC4 domains have been implicated in interactions with other CC4 domains, so we hypothesized that they might mediate SNX5-CHC22 binding. This was investigated by expressing smaller fragments of each partner protein in bait and prey vectors (Figure 7, A and B). When the N-terminal SNX5 CC4 domain was deleted from the SNX5 fragment, interaction with CHC22 Hub was abrogated. Although a fragment com- posed of that N-terminal CC4 domain alone (259-289) did not interact with $\mathrm{CHC} 22 \mathrm{Hub}$, a slightly larger fragment extending 20 residues to either side (239-309) did interact with $\mathrm{CHC} 22 \mathrm{Hub}$, suggesting that this domain does mediate SNX5-CHC22 binding but needs flanking residues to fold properly. Removal of the CC4 domain in CHC22 also abrogated the SNX5-CHC22 interaction. However for CHC22 a much longer fragment (CHC22 1267-1522), albeit still comprising the $\mathrm{CC} 4$ domain, was required for detecting an interaction with SNX5. Notably, the CC4 domain in CHC22 overlaps with the LC-binding site in the equivalent region of $\mathrm{CHC17}$. Although an interaction between $\mathrm{CHC} 22$ and the fragment of LCb (residues 59-212) comprising the CHC17 binding site (Chen et al., 2002) can be detected by yeast two hybrid assay (Figure 7B), we have previously reported that more sensitive two-hybrid assays reveal that $\mathrm{CHC} 17$ binds more strongly than CHC22 to LCb (Liu et al., 2001). Here we further demonstrate that $\mathrm{CHC} 22-\mathrm{LCb}$ binding was more sensitive to protein truncation than the CHC17-LCb binding (Figure 7B). This correlates with our published observations that although CHC17 binding to LCs is detected in cells by colocalization and coimmunoprecipitation and by protein purification after coexpression (Liu et al., 2001), CHC22 binding to LCs is not detected in any of these protein assays. Thus, CHC22 might have the capacity to bind LCs weakly, 

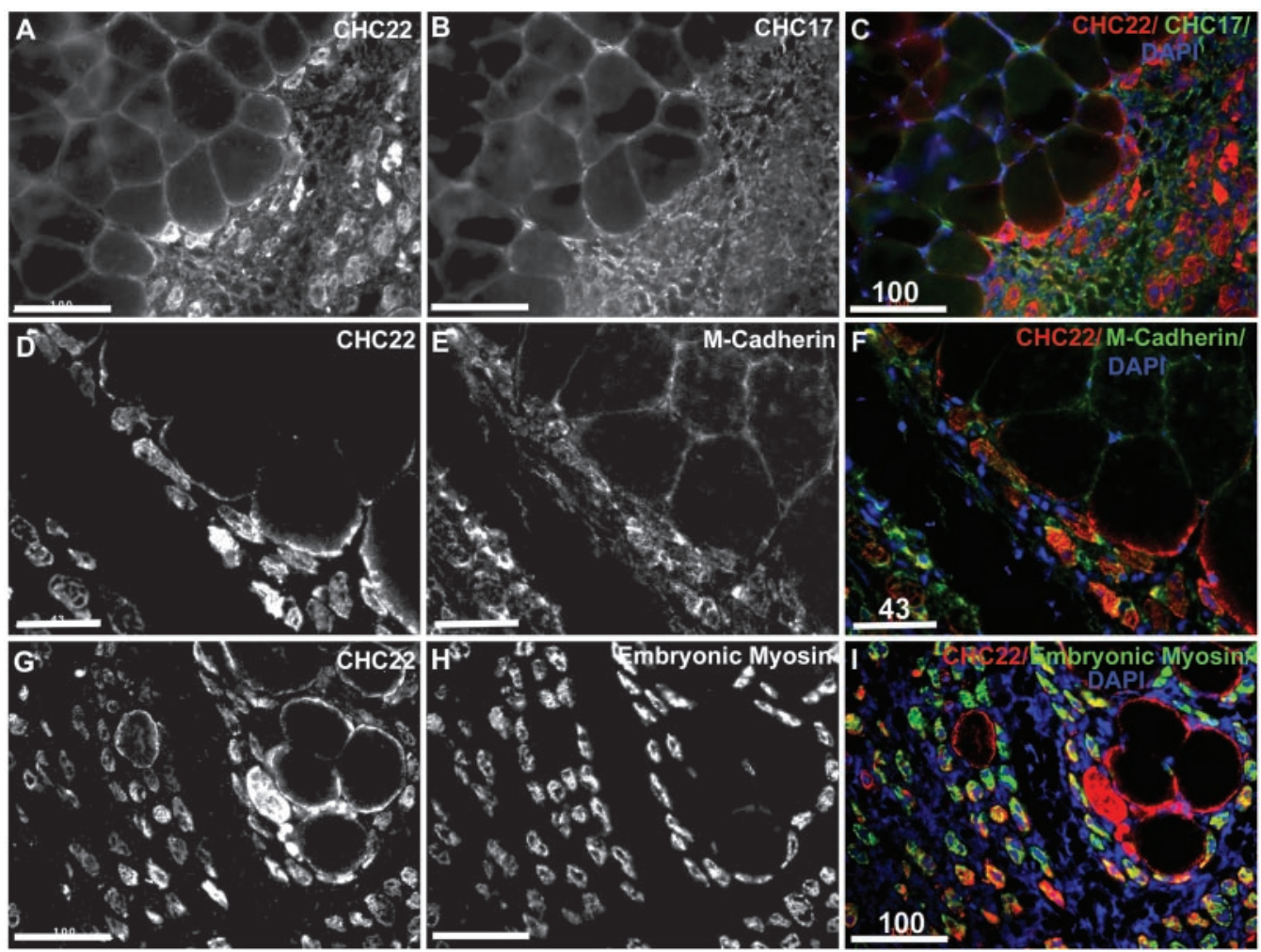

Figure 6. $\mathrm{CHC} 22$ expression localizes to regenerating fibers after cardiotoxin injection. Transverse sections of rat tibialis anterior muscle collected on day 3 after injection of cardiotoxin venom (Hoshino et al., 2002a, 2002b, 2002c). Samples were double labeled for CHC22 (A and $\mathrm{C}$, red; D and F, red; G and I, red) and for CHC17 (B and C, green), M-cadherin (E and F, green), or embryonic myosin (H and I, green). The regenerating fibers were characterized by centrally localized nuclei, visualized by DAPI staining in the merged panels (C, F, and I, blue). Each row shows the same field viewed with different filters and the right hand panel shows the merged images. Scale bars in micrometers

but this is not observed at the protein level and furthermore, the potential binding site for LCs is the SNX5 binding site, so would be occupied in cells expressing SNX5.

The interaction between SNX5 and CHC22 was confirmed at the protein level by coimmunoprecipitation from cells expressing both proteins. Permanently transfected HeLa cells that express full-length $\mathrm{CHC} 22$ or $\mathrm{CHC} 22 \mathrm{Hub}$ on induction with doxycycline (Liu et al., 2001) also express detectable levels of these proteins without induction, due to leaky promoters. These cells were transiently transfected with full-length SNX5 with a three-epitope FLAG tag attached to its N-terminus (no antibody specific for native SNX5 is available). FLAG-tagged SNX5 coimmunoprecipitated with both full-length and Hub CHC22- compared with mock-transfected cells (Figure 7C). The SNX5-FLAG protein was not isolated in nonspecific immunoprecipitates using rabbit preimmune serum (unpublished data). These transfection experiments indicate that binding between $\mathrm{CHC} 22$ and SNX5 occurs in cells and suggest that SNX5 is a likely partner for $\mathrm{CHC} 22$ in muscle, where both are expressed at high levels. This interaction was shown to be specific for $\mathrm{CHC} 22$ vs. CHC17 (Figure 7D). In this case the endogenous heavy chains were immunoprecipitated from HeLa cells and incubated with lysate from cells transfected with FLAG tagged SNX5. The expressed SNX5 bound only to the immunoisolated $\mathrm{CHC} 22$ and not to the immunoisolated CHC17. This experiment also indicates that even when CHC22 and SNX5 are not coexpressed in the same cell, binding between them is sufficiently avid that it can be detected. The interaction of SNX5 with the CHC22 domain that corresponds to the LC-binding domain of CHC17 suggests a mechanism by which $\mathrm{CHC} 22$ could be regulated differently from $\mathrm{CHC} 17$ and further suggests that SNX5 could serve as a membrane adaptor for CHC22.

\section{DISCUSSION}

We report here the first characterization of the CHC22 isoform of clathrin in skeletal muscle. CHC22 was found to be concentrated at NMJs and MTJs in human and rat skeletal muscle. Its expression was increased during myogenesis and in regenerating muscle. This general pattern of $\mathrm{CHC} 22$ expression, including its polarization during myogenesis, suggests a role for $\mathrm{CHC} 22$ at regions of the sarcolemma that are involved in contact with ECM. Such regions are fundamental to muscle function and are vulnerable targets for hereditary muscle diseases. Morphological analysis of CHC22 further established its distinct distribution and function relative to the ubiquitous $\mathrm{CHC} 17$ clathrin. Our observation that SNX5 binds CHC22 suggests a mechanism by which their functions segregate and also implicates SNX5 in muscle membrane traffic. These findings demonstrate that a novel clathrin and a novel adaptor can contribute to membrane traffic specialization in muscle tissue.

\section{Distinct Regulation, Distribution, and Function of CHC22} Relative to $\mathrm{CHC17}$

A recurring observation throughout this study is that CHC22 and CHC17 have consistently distinct intracellular localizations. The function of $\mathrm{CHC} 17$ is well characterized in 
A
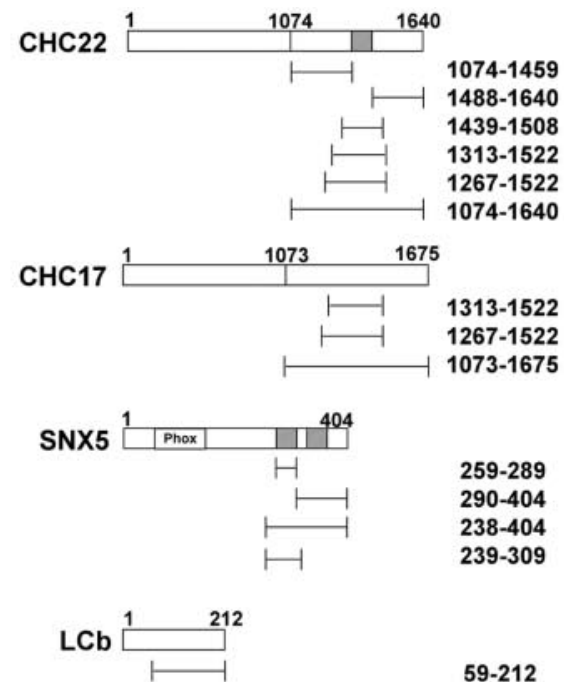

$59-212$

\begin{tabular}{|c|c|c|}
\hline $\begin{array}{l}\text { SNX5 Constructs in } \\
\text { prey vector pACT2 }\end{array}$ & $\begin{array}{l}\text { Interaction with CHC22 } \\
\text { Hub 1074-1640 in bait } \\
\text { vector pGBT9 }\end{array}$ & $\begin{array}{l}\text { Interaction with CHC17 } \\
\text { Hub 1073-1675 in bait } \\
\text { vector pGBT9 }\end{array}$ \\
\hline $259-289$ & - & - \\
\hline $290-404$ & - & - \\
\hline $238-404$ & + & - \\
\hline $239-309$ & + & - \\
\hline $\begin{array}{l}\text { CHC22 Constructs in } \\
\text { bait vector pGBT9 }\end{array}$ & $\begin{array}{l}\text { Interaction with SNX5 } \\
\text { 238-404 in prey vector } \\
\text { pACT2 }\end{array}$ & $\begin{array}{c}\text { Interaction with LCb 59- } \\
\text { 212 in prey vector pACT2 }\end{array}$ \\
\hline $1074-1459$ & - & - \\
\hline $1488-1640$ & - & + \\
\hline $1439-1508$ & - & - \\
\hline $1313-1522$ & - & - \\
\hline $1267-1522$ & + & + (low) \\
\hline $1074-1640(H u b)$ & - & + \\
\hline $\begin{array}{l}\text { CHC17 Constructs in } \\
\text { bait vector pGBT9 }\end{array}$ & $\begin{array}{l}\text { Interaction with SNX5 } \\
238-404 \text { in prey vector } \\
\text { pACT2 }\end{array}$ & $\begin{array}{c}\text { Interaction with LCb 59- } \\
212 \text { in prey vector pACT2 }\end{array}$ \\
\hline $1313-1522$ & - & + \\
\hline $1267-1522$ & - & + \\
\hline $1073-1675(H u b)$ & - & + \\
\hline
\end{tabular}
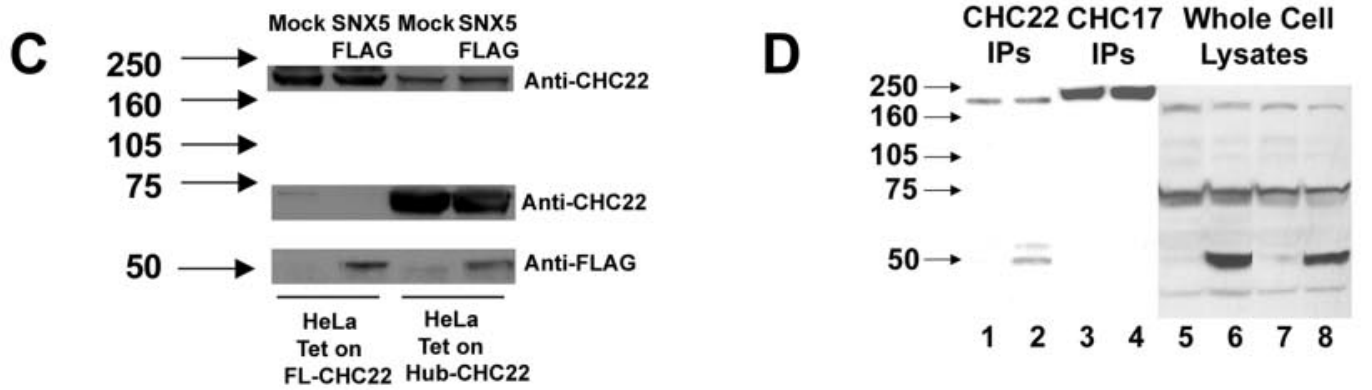

Figure 7. 
organelle biogenesis and receptor-mediated endocytosis (reviewed in Brodsky et al., 2001). Because CHC22 does not colocalize with any proteins associated with these conventional clathrin pathways, its function in muscle appears to be entirely different from that of $\mathrm{CHC} 17$. These conventional CCV functions in muscle are most likely still carried out by $\mathrm{CHC} 17$. The dramatic difference in distribution and expression levels of these two clathrin heavy chains is surprising given the similarity in protein sequence $(85 \%)$. On sequence comparison of $\mathrm{CHC} 22$ and $\mathrm{CHC} 17$ it is difficult to pinpoint functional domains, because the differences in protein sequence are dispersed throughout the entire protein. We have previously shown that $\mathrm{CHC} 22$ trimerizes as does $\mathrm{CHC} 17$, but that it does not interact with the clathrin LCs, as purified proteins or in cells. Here we show that SNX5 binds to the region in $\mathrm{CHC} 22$ where the light chain would normally bind to $\mathrm{CHC} 17$ for negative regulation of clathrin assembly (Ungewickell and Ungewickell, 1991; Liu et al., 1995). This region has a predicted CC4 domain in $\mathrm{CHC} 22$ that is not predicted for CHC17 and SNX5 did not interact with $\mathrm{CHC} 17$. We propose that this CC4 domain mediates the interaction between $\mathrm{CHC} 22$ and $\mathrm{SNX} 5$ because the partner domain in SNX5 is the N-terminal CC4 domain present in SNX5, which has two CC4 domains. SNX proteins are predicted to heterodimerize or homodimerize through their CC4 domains. If the N-terminal CC4 domain in SNX5 binds $\mathrm{CHC} 22$, the C-terminal CC4 domain could still bind other proteins. One candidate is SNX1, which was recently shown to interact with SNX5 (R. Teasdale and P. Gleeson, unpublished results). Yeast two-hybrid analysis indicated that CHC22 could potentially bind to clathrin LCs in the SNX5binding region. On the basis of the lack of detectable binding between $\mathrm{CHC} 22$ protein and LCs in vitro and their lack of

Figure 7 (facing page). Sorting nexin 5 interacts specifically with $\mathrm{CHC} 22$ through a coiled-coil 4 domain. (A) Diagrams indicating the domains of $\mathrm{CHC} 22, \mathrm{CHC} 17, \mathrm{SNX} 5$, and clathrin light-chain LCb that were tested as bait or prey in yeast two-hybrid experiments reported in B. The fragments tested are delineated below the diagram of each full-length protein. The shaded regions indicate predicted coiled-coil 4 (CC4) domains in CHC22 and SNX5 that are absent in $\mathrm{CHC} 17$ and LCb sequences. (B) Two-hybrid assays were performed with the constructs shown in A cloned into the bait vector (pGBT9) or prey vector (pACT2), as indicated. Interactions were measured by plate growth assay and are indicated as $(+)$ or $(-)$. Attenuated growth was observed with those combinations marked "low." (C) Permanently transfected HeLa cells expressing full-length CHC22 (HeLa-Tet on-CHC22) or CHC22 Hub fragment (1074-1640; HeLaTet on-CHC22 Hub; Liu et al., 2001) were transfected with fulllength SNX5 with a FLAG tag at the N-terminus (SNX5-FLAG) or exposed to transfection conditions without DNA (Mock). After $16 \mathrm{~h}$ cell lysates were prepared, CHC22 was immunoprecipitated with specific polyclonal antibody, and immunoprecipitates were analyzed by immunoblotting. $\mathrm{CHC} 22$ and $\mathrm{CHC} 22-\mathrm{Hub}$ fragment were detected with polyclonal anti-CHC22 and the coprecipitating SNX5 was detected with $\mathrm{mAb}$ to the FLAG epitope. Some endogenous full-length CHC22 is detected in HeLa cells expressing CHC22 Hub. (D) Clathrin heavy-chains $\mathrm{CHC} 22$ or $\mathrm{CHC} 17$ were immunoisolated using specific polyclonal antibodies from HeLa cell lysates then incubated with lysates of mock-transfected cells (lanes 1,3,5, and 7) or lysates of cells expressing SNX5-FLAG (lanes 2, 4, 6, and 8). Immunoisolated $\mathrm{CHC}$ were detected by separately immunoblotting the top half of lanes 1 and 2 or 3 and 4 with monoclonal antibodies specific for $\mathrm{CHC} 22$ or $\mathrm{CHC} 17$, respectively. FLAGtagged SNX5 bound to CHC22 (lane 2) but not CHC17 (lane 4) was detected by blotting the bottom half of lanes 1-4 with monoclonal against the FLAG epitope. The whole cell lysates that were used as a source of SNX5-FLAG or as control mock-transfected lysates are shown in lanes 5-8 blotted for FLAG-tagged SNX5. colocalization with $\mathrm{CHC} 22$ in vivo, we suggest that SNX5 occupies the potential LC binding site with the resulting effect that $\mathrm{CHC} 22$ is regulated differently than $\mathrm{CHC} 17$.

SNX5 is one of a subgroup of the SNX family that have a 70-110-residue SNX-PX domain and a C-terminal domain with multiple CC4 domains (Teasdale et al., 2001). Both the PX and the coiled-coil domains have been shown independently to localize SNX5 to endosomal structures (Teasdale et al., 2001). Membrane recruitment may also be mediated through their interaction with cargo. Thus SNX5 could potentially serve as a membrane adaptor for CHC22. In muscle and in myoblasts, $\mathrm{CHC} 22$ did not colocalize with the adaptors that normally bind $\mathrm{CHC} 17$ (AP1, AP2, AP3, or GGA1). In HeLa cells, we previously showed a potential interaction between $\mathrm{CHC} 22$ and $\mathrm{AP} 1$ and $\mathrm{AP} 3$ but not AP2. We speculate that in muscle the AP-binding site might be obscured by another binding protein so that membrane associations are mediated differently, possibly through SNX5. Preliminary yeast two-hybrid searches for proteins that specifically bind the terminal domain of $\mathrm{CHC} 22$, which binds adaptors in $\mathrm{CHC} 17$, revealed a number of potential binding candidates to be explored. These findings and additional yeast twohybrid searches with the $\mathrm{CHC} 22$ Hub suggest that additional tissue-specific regulatory proteins that control the specialized function of $\mathrm{CHC} 22$ are likely to emerge.

\section{Roles for CHC22 at Specialized Regions of the Sarcolemma}

Various specialized membrane regions and membrane transport pathways are present in skeletal muscle (for review, see Towler et al., 2004). These include the sarcolemma, sarcoplasmic reticulum, T-tubules, NMJs, MTJs, costameres, GLUT4 transport, and membrane fusion. Many muscle specific isoforms of proteins exist allowing these systems to function in skeletal muscle. For example, caveolin 3 has been implicated in T-tubule development (Parton et al., 1997). CHC22 did not localize to T-tubules and does not colocalize with caveolin 3 in primary rat myofibers or in muscle tissue; therefore, a role in T-tubule biogenesis or maintenance was ruled out. Similarly CHC22 did not colocalize with GLUT4 markers (Table 1). However its concentration at NMJs (Figures 2 and 5) and at MTJs (Figures 2 and 3) is evident.

NMJs are the site where the motor nerve innervates muscle. Membrane traffic is polarized here and subneural nuclei become specialized upon maturation of the NMJ to initiate transcription of locally required proteins. These proteins are in turn transported to their sites of action and CHC22 may have a role in these specific transport pathways. The process of innervation stimulates the localization of some proteins to NMJs and it can also influence membrane traffic at NMJs (Antony et al., 1995). The pattern of CHC22 distribution in regenerating muscle was consistent with its localization being controlled by innervation. This could be the first example of a membrane traffic protein being regulated in such a way in skeletal muscle and would enable a role in specialized local protein transport. Membrane traffic at MTJs has to respond to mechanical stress to mediate membrane repair, thus MTJs also require stimulated localized membrane transport, similar to that which occurs at NMJs and CHC22 could be involved in such pathways.

An alternative and not necessarily exclusive hypothesis regarding $\mathrm{CHC} 22$ function can be proposed based on its concentration at NMJs and MTJs and its partial colocalization with desmin, nestin, and dystrophin. NMJs, MTJs, and costameres (the sites where Z-disks meet the sarcolemma) all represent organized regions of sarcolemma where muscle contacts ECM proteins. The dystrophin/dystroglycan com- 
plex is concentrated at these sites as well as integrin $\alpha 7 \beta 1$ (Clark et al., 2002). On the cytoplasmic side of the sarcolemma, these ECM contact molecules have extensive interactions with adaptor and signaling proteins that are associated with a scaffold of intermediate filament proteins (Michele and Campbell, 2003). Desmin is localized to all three ECM contact regions and nestin, a desmin-binding protein, concentrates more specifically at NMJs and MTJs (Carlsson et al., 1999; Vaittinen et al., 1999; Clark et al., 2002). The partial localization of CHC22 with desmin and nestin both during myogenesis and in mature skeletal muscle suggests that $\mathrm{CHC} 22$ might also have a scaffold function or somehow contribute to the supramolecular organization of these sites of ECM contact. The dramatic localization of $\mathrm{CHC} 22$ to the poles of fused myoblasts, where they interact with the culture dish, is also consistent with an organizational role at ECM contact sites.

Thus, our morphological analysis of $\mathrm{CHC} 22$ distribution suggests it could either be involved in specialized protein transport pathways that are shared by NMJs and MTJs or could be involved in organizing proteins at these sites of ECM contact. The intramuscular staining pattern of $\mathrm{CHC} 22$ is consistent with vesicular structures, and its association with SNX5 is consistent with a role in membrane traffic. On the other hand, the fibrous appearance of $\mathrm{CHC} 22$ staining at the NMJ, suggests a potential structural role, as well. If CHC22 self-assembles in a manner analogous to $\mathrm{CHC17}$, it could certainly play a role in membrane protein organization as well as vesicle coat formation.

\section{Predicted Roles for CHC22 in Muscle Regeneration and Muscle Disease}

The increase of $\mathrm{CHC} 22$ expression during muscle regeneration suggests a role in reestablishing muscle function. Regeneration involves reformation of contacts with the ECM both at sites of reinnervation and at muscle reattachment sites. The potential involvement of $\mathrm{CHC} 22$ in transport to sites of muscle membrane stress at MTJs is also consistent with its upregulation during regeneration. We have noted the presence of myoD-binding sites upstream of the $\mathrm{CHC} 22$ gene (Towler and Brodsky, unpublished results). MyoDmediated transcription is essential both in myogenesis and repair and the fact that $\mathrm{CHC} 22$ expression has the potential to respond to this transcription factor supports its participation in these muscle functions. Further studies of $\mathrm{CHC} 22$ expression and regulation will contribute to our understanding of muscle regeneration and stimulation of its expression may contribute to therapeutic approaches to muscle disease.

\section{ACKNOWLEDGMENTS}

We thank Chih-ying Chen from the Brodsky lab for control constructs for the yeast two-hybrid experiments and for sharing the skeletal muscle cDNA library from Clontech that she amplified, Tristam Parslow and Kimberly Topp for donating muscle samples from UCSF pathology archives, and Stephen Kaufman for the L8E63 cell line and an antibody to $\alpha 7$-Integrin. We thank the following investigators for antibodies (listed in MATERIALS AND METHODS): Robert Edwards, Margaret S. Robinson, Georgine Faulkner, Regis Kelly, Juan Bonifacino, and Jeffrey Pessin. The mAb against embryonic myosin (F1.652) developed by Helen M. Blau was obtained from the Developmental Studies Hybridoma Bank developed under the auspices of the National Institute of Child Health and Human Development and maintained by the University of Iowa, Department of Biological Sciences, Iowa City, IA. We also thank Christine Knuehl, Sherri Newmyer, and Chih-ying Chen for technical advice and members of the Ordahl lab for their input and encouragement throughout the project. M.C.T. is a Wellcome Prize Traveling Fellow and was funded by the Muscular Dystrophy Association for part of this work. This research was supported by National Institutes of Health Grants GM057657 and GM038093 to F.M.B. C.O. is supported by grant number RO1
AR44483. P.G. and R.G.P. are supported by grants from the Australian National Health and Medical Research Council.

\section{REFERENCES}

Acton, S., and Brodsky, F.M. (1990). Predominance of clathrin light chain LCb correlates with the presence of a regulated secretory pathway. J. Cell Biol. 111, 1419-1426

Antony, C., Huchet, M., Changeux, J.P., and Cartaud, J. (1995). Developmental regulation of membrane traffic organization during synaptogenesis in mouse diaphragm muscle. J. Cell Biol. 130, 959-968.

Blank, G.S., and Brodsky, F.M. (1986). Site-specific disruption of clathrin assembly produces novel structures. EMBO J. 5, 2087-2095.

Brodsky, F.M., Chen, C.Y., Knuehl, C., Towler, M.C., and Wakeham, D.E. (2001). Biological basket weaving: Formation and function of clathrin-coated vesicles. Annu. Rev. Cell Dev. Biol. 17, 517-568

Burkin, D.J., and Kaufman, S.J. (1999). The alpha7beta1 integrin in muscle development and disease. Cell Tissue Res. 296, 183-190.

Carlsson, L., Li, Z., Paulin, D., and Thornell, L.E. (1999). Nestin is expressed during development and in myotendinous and neuromuscular junctions in wild type and desmin knock-out mice. Exp. Cell Res. 251, 213-223.

Chen, C.Y., Reese, M.L., Hwang, P.K., Ota, N., Agard, D., and Brodsky, F.M (2002). Clathrin light and heavy chain interface: alpha-helix binding superhelix loops via critical tryptophans. EMBO J. 21, 6072-6082.

Clark, K.A., McElhinny, A.S., Beckerle, M.C., and Gregorio, C.C. (2002). Striated muscle cytoarchitecture: an intricate web of form and function. Annu. Rev. Cell. Dev. Biol. 18, 637-706

Cozier, G.E., Carlton, J., McGregor, A.H., Gleeson, P.A., Teasdale, R.D., Mellor, H., and Cullen, P.J. (2002). The phox homology (PX) domain-dependent, 3-phosphoinositide-mediated association of sorting nexin-1 with an early sorting endosomal compartment is required for its ability to regulate epidermal growth factor receptor degradation. J. Biol. Chem. 277, 4873048736.

Ervasti, J.M. (2003). Costameres: the Achilles' heel of Herculean muscle. J. Biol. Chem. 278, 13591-13594.

Fischer, D., Schroers, A., Blumcke, I., Urbach, H., Zerres, K., Mortier, W., Vorgerd, M., and Schroder, R. (2003). Consequences of a novel caveolin-3 mutation in a large German family. Ann. Neurol. 53, 233-241.

Holmes, S.E. et al. (1997). Disruption of the clathrin heavy chain-like gene (CLTCL) associated with features of DGS/VCFS: a balanced $(21 ; 22)(\mathrm{p} 12 ; \mathrm{q} 11)$ translocation. Hum. Mol. Genet. 6, 357-367.

Hoshino, S., Ohkoshi, N., Ishii, A., Kameya, S., Takeda, S., and Shoji, S. (2001) The expression of dystrophin and alpha1-syntrophin during skeletal muscle regeneration. J. Muscle Res. Cell Motil. 22, 185-191.

Hoshino, S., Ohkoshi, N., Ishii, A., and Shoji, S. (2002a). The expression of alpha-dystrobrevin and dystrophin during skeletal muscle regeneration. J. Muscle. Res. Cell Motil. 23, 131-138.

Hoshino, S., Ohkoshi, N., Ishii, A., and Shoji, S. (2002b). The expression of dystrophin, alpha-sarcoglycan, and beta-dystroglycan during skeletal muscle regeneration: immunohistochemical and western blot studies. Acta Histochem. 104, 139-147.

Hoshino, S., Ohkoshi, N., Ishii, A., and Shoji, S. (2002c). The expression of neuronal nitric oxide synthase and dystrophin in rat regenerating muscles. J. Muscle Res. Cell Motil. 23, 139-145.

Kaisto, T., Rahkila, P., Marjomaki, V., Parton, R.G., and Metsikko, K. (1999). Endocytosis in skeletal muscle fibers. Exp. Cell Res. 253, 551-560.

Karsch-Mizrachi, I., Travis, M., Blau, H., and Leinwand, L.A. (1989). Expression and DNA sequence analysis of a human embryonic skeletal muscle myosin heavy chain gene. Nucleic Acids Res. 17, 6167-6179.

Kaufman, S.J., Parks, C.M., Bohn, J., and Faiman, L.E. (1980). Transformation is an alternative to normal skeletal muscle development. Exp. Cell Res. 125, 333-349.

Kedra, D., Peyrard, M., Fransson, I., Collins, J.E., Dunham, I., Roe, B.A., and Dumanski, J.P. (1996). Characterization of a second human clathrin heavy chain polypeptide gene (CHL-22) from chromosome 22q11. Hum. Mol. Genet. $5,625-631$

Kubisch, C. et al. (2003). Homozygous mutations in caveolin-3 cause a severe form of rippling muscle disease. Ann. Neurol. 53, 512-520.

Liu, S.-H., Towler, M.C., Chen, E., Chen, C.-Y., Song, W., Apodaca, G., and Brodsky, F.M. (2001). A novel clathrin homolog that co-distributes with cytoskeletal components functions in the trans-Golgi network. EMBO J. 20, 272-284. 
Liu, S.-H., Wong, M.L., Craik, C.S., and Brodsky, F.M. (1995). Regulation of clathrin assembly and trimerization defined using recombinant triskelion hubs. Cell 83, 257-267.

Long, K.R., Trofatter, J.A., Ramesh, V., McCormick, M.K., and Buckler, A.J. (1996). Cloning and characterization of a novel human clathrin heavy chain gene (CLTCL). Genomics 35, 466-472.

Matsuda, R., Spector, D.H., and Strohman, R.C. (1983). Regenerating adult chicken skeletal muscle and satellite cell cultures express embryonic patterns of myosin and tropomyosin isoforms. Dev. Biol. 100, 478-488.

Michele, D.E., and Campbell, K.P. (2003). Dystrophin-glycoprotein complex: post-translational processing and dystroglycan function. J. Biol. Chem. 278, 15457-15460.

Muller, A.J. et al. (2003). Targeted disruption of the murine Bin1/Amphiphysin II gene does not disable endocytosis but results in embryonic cardiomyopathy with aberrant myofibril formation. Mol. Cell. Biol. 23, 4295-4306.

Nathke, I., Hill, B.L., Parham, P., and Brodsky, F.M. (1990). The calciumbinding site of clathrin light chains. J. Biol. Chem. 265, 18621-18627.

Otsuki, T., Kajigaya, S., Ozawa, K., and Liu, J.M. (1999). SNX5, a new member of the sorting nexin family, binds to the Fanconi anemia complementation group A protein. Biochem. Biophys. Res. Commun. 265, 630-635.

Parton, R.G., Way, M., Zorzi, N., and Stang, E. (1997). Caveolin-3 associates with developing T-tubules during muscle differentiation. J. Cell Biol. 136, 137-154.

Ralston, E., Lu, Z., and Ploug, T. (1999). The organization of the Golgi complex and microtubules in skeletal muscle is fiber type-dependent. J. Neurosci. 19, 10694-10705.

Simpson, F., Whitehead, J.P., and James, D.E. (2001). GLUT4 - at the cross roads between membrane trafficking and signal transduction. Traffic 2, 2-11.
Sirotkin, H. et al. (1996). Isolation of a new clathrin heavy chain gene with muscle-specific expression from the region commonly deleted in velo-cardiofacial syndrome. Hum. Mol. Genet. 5, 617-624.

Teasdale, R.D., Loci, D., Houghton, F., Karlsson, L., and Gleeson, P.A. (2001) A large family of endosome-localized proteins related to sorting nexin 1. Biochem. J. 358, 7-16.

Topp, K.S. et al. (2003). Alterations in skeletal muscle structure are minimized with steroid withdrawal after renal transplantation. Transplantation 76, 667673.

Towler, M.C., Kaufman, S.J., and Brodsky, F.M. (2004). Membrane traffic in skeletal muscle. Traffic 5, 129-139.

Ungewickell, E., and Ungewickell, H. (1991). Bovine brain clathrin light chains impede heavy chain assembly in vitro. J. Biol. Chem. 266, 12710-12714

Vaittinen, S., Lukka, R., Sahlgren, C., Rantanen, J., Hurme, T., Lendahl, U., Eriksson, J.E., and Kalimo, H. (1999). Specific and innervation-regulated expression of the intermediate filament protein nestin at neuromuscular and myotendinous junctions in skeletal muscle. Am. J. Pathol. 154, 591-600.

Worby, C.A., and Dixon, J.E. (2002). Sorting out the cellular functions of sorting nexins. Nat. Rev. Mol. Cell. Biol. 3, 919-931.

Xu, Y., Hortsman, H., Seet, L., Wong, S.H., and Hong, W. (2001). SNX3 regulates endosomal function through its $\mathrm{PX}$-domain-mediated interaction with PtdIns(3)P. Nat. Cell Biol. 3, 658-666.

Yaffe, D., and Saxel, O. (1977). A myogenic cell line with altered serum requirements for differentiation. Differentiation 7, 159-166.

Zhang, B., and Zelhof, A.C. (2002). Amphiphysins: raising the BAR for synaptic vesicle recycling and membrane dynamics. Traffic 3, 452-460. 